\title{
Pyruvate kinase isoform M2 impairs cognition in systemic lupus erythematosus by promoting microglial synaptic pruning via the $\beta$-catenin signaling pathway
}

$\mathrm{Li} \mathrm{Lu}^{1,2+}$, Hailin Wang ${ }^{1,2+}$, Xuan Liu ${ }^{1,2}$, Liping Tan ${ }^{1,2}$, Xiaoyue Qiao ${ }^{1,2}$, Jiali Ni ${ }^{1,2}$, Yang Sun ${ }^{4^{*}}$, Jun Liang ${ }^{3^{*}}$, Yayi Hou ${ }^{1,2,3^{*}}$ and Huan Dou ${ }^{1,2,3^{*}}$ (1)

\begin{abstract}
Background: Neuropsychiatric systemic lupus erythematosus (NPSLE) is a severe complication, which involves pathological damage to the brain and cognitive function. However, its exact mechanism of action still remains unclear. In this study, we explored the role of microglia in the cognitive dysfunction of NPSLE mice. We also analyzed and compared the metabolites in the hippocampal tissues of the lupus model and control mice.

Methods: MRL/MpJ-Fas ${ }^{\mathrm{lpr}}$ (MRL/lpr) female mice were used as the NPSLE mouse model. Metabolomics was used to assess hippocampal glycolysis levels. Glucose, lactic acid, IL-6, and IL-1 $\beta$ of the hippocampus were detected by ELISA. Based on the glycolysis pathway, we found that pyruvate kinase isoform M2 (PKM2) in the hippocampus was significantly increased. Thus, the expression of PKM2 was detected by qRT-PCR and Western blotting, and the localization of

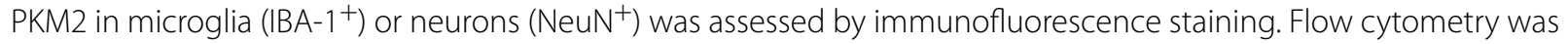
used to detect the number and phenotype of microglia; the changes in microglial phagocytosis and the $\beta$-catenin signaling pathway were detected in BV2 cells overexpressing PKM2. For in vivo experiments, MRL/Ipr mice were treated with AAV9-shPKM2. After 2 months, Morris water maze and conditional fear tests were applied to investigate the cognitive ability of mice; H\&E and immunofluorescence staining were used to evaluate brain damage; flow cytometry was used to detect the phenotype and function of microglia; neuronal synapse damage was monitored by qRT-PCR, Western blotting, and immunofluorescence staining.
\end{abstract}

Results: Glycolysis was elevated in the hippocampus of MRL/lpr lupus mice, accompanied by increased glucose consumption and lactate production. Furthermore, the activation of PKM2 in hippocampal microglia was observed in

\footnotetext{
*Correspondence: yangsun@nju.edu.cn; 13505193169@139.com;

yayihou@nju.edu.cn; douhuan@nju.edu.cn

${ }^{\dagger} \mathrm{Li}$ Lu and Hailin Wang contributed equally to this article

${ }^{1}$ The State Key Laboratory of Pharmaceutical Biotechnology, Division

of Immunology, Medical School, Nanjing University, Nanjing 210093,

People's Republic of China

${ }^{3}$ Department of Rheumatology and Immunology, Nanjing Drum Tower

Hospital, The Affiliated Hospital of Nanjing University Medical School,

Nanjing 210008, People's Republic of China

${ }^{4}$ The State Key Laboratory of Pharmaceutical Biotechnology

and Collaborative Innovation Center of Chemistry for Life Sciences,

School of Life Sciences, Nanjing University, Nanjing 210023, China

Full list of author information is available at the end of the article
}

(c) The Author(s) 2021. Open Access This article is licensed under a Creative Commons Attribution 4.0 International License, which permits use, sharing, adaptation, distribution and reproduction in any medium or format, as long as you give appropriate credit to the original author(s) and the source, provide a link to the Creative Commons licence, and indicate if changes were made. The images or other third party material in this article are included in the article's Creative Commons licence, unless indicated otherwise in a credit line to the material. If material is not included in the article's Creative Commons licence and your intended use is not permitted by statutory regulation or exceeds the permitted use, you will need to obtain permission directly from the copyright holder. To view a copy of this licence, visit http://creativecommons.org/licenses/by/4.0/. The Creative Commons Public Domain Dedication waiver (http://creativeco mmons.org/publicdomain/zero/1.0/) applies to the data made available in this article, unless otherwise stated in a credit line to the data. 
lupus mice. Cell experiments showed that PKM2 facilitated microglial activation and over-activated microglial phagocytosis via the $\beta$-catenin signaling pathway. In vivo, AAV9-shPKM2-treated mice showed decreased microglial activation and reduced neuronal synapses loss by blocking the $\beta$-catenin signaling pathway. Furthermore, the cognitive impairment and brain damage of MRL/Ipr mice were significantly relieved after microglial PKM2 inhibition.

Conclusion: These data indicate that microglial PKM2 have potential to become a novel therapeutic target for treating lupus encephalopathy.

Keywords: Systemic lupus erythematosus encephalopathy, Aerobic glycolysis, Pyruvate kinase isoform M2, Microglia, Phagocytosis, LC-MS analysis

\section{Introduction}

Systemic lupus erythematosus (SLE) is a complex autoimmune disease affecting various organs in the body $[1$, 2]. Clinically, 12-95\% of SLE patients develop psychiatric and neurologic manifestations, also known as neuropsychiatric systemic lupus erythematosus (NPSLE), which severely affects life quality and increases diseaserelated mortality [3]. Among the 19 NPSLE syndromes defined by the American College of Rheumatology (ACR) [4], cognitive dysfunction has been identified as one of the most distressing symptoms in SLE [5]. It often develops insidiously, and presents and progresses independently. Also, it usually does not respond to standard immunosuppression [6, 7]. Accumulating evidence indicates that complex and interconnected mechanisms promote the development of SLE-related cognitive impairment; however, the specific pathogenesis is still unclear [3].

Brain function is correlated to an adequate metabolic cost, accounting for $20 \%$ of the whole-body energy consumption in humans $[8,9]$. In 2000, positron emission tomography (PET) demonstrated that lupus cerebritis was positively associated with the significant alterations in glucose metabolism with regional specificity in the brains of SLE patients [10,11]. Furthermore, an increase in total brain glutamine, glutamate, and lactate concentrations has been found in MRL/lpr lupus mouse model compared to the $\mathrm{MRL}^{+/+}$control mice by ${ }^{1} \mathrm{H}$ and ${ }^{13} \mathrm{C}$ nuclear magnetic resonance (NMR) spectroscopy detection [12]. Moreover, metabolic changes were observed in specific brain regions in SLE patients with cognitive impairment by multimodal magnetic resonance imaging (MRI) [13]. Interestingly, aging brains showed high lactate levels and were susceptible to several neurodegenerative diseases accompanied by metabolic alterations, contributing to cognitive impairment [14]. In anti-DNA antibodies $(\text { DNRAbs })^{+}$mice, significant metabolic changes and spatial memory impairment were observed with neuronal loss in hippocampal regions [15]. Taken together, these studies suggest that the regional metabolic abnormalities are critical neuropathological features on the cognitive manifestation in NPSLE.
Pyruvate kinase isoform M2 (PKM2), a crucial mediator of cellular energetics, catalyzes the conversion of phosphoenolpyruvate to pyruvate during glycolysis and is identified as the hub network protein for the regulation of idiopathic autism [10]. Furthermore, a proteomics-based approach revealed that PKM2 is one of the main differential proteins involved in the pathogenesis of infantile spasms with severe cognitive dysfunction [11]. Propofolprotective effects on ketamine-induced neonatal cognition damage have been linked to PKM2 expression in the hippocampus [16]. Moreover, PKM2 was found to be significantly oxidized in the hippocampi of mild cognitive impairment patients and functionally involved in energy metabolism and synaptic plasticity [17]. Besides, methamphetamine-induced neurocognitive deficits exhibited PKM2 activity impairment [18]. Nonetheless, PKM2 in cognitive dysfunction and progression of NPSLE remains poorly understood.

Microglia, the tissue-resident macrophages of the central nervous system (CNS), have a major role in brain homeostasis $[19,20]$. In addition to their intended role in host defense, microglia continuously stretch, retract, and restructure to monitor the functional status of their surroundings and remove the accumulated metabolites or cell debris to maintain brain homeostasis [21-23]. In the adult brain, microglia regulate advanced cognitive functions, such as learning and memory [24, 25]. When homeostasis is altered, or the brain tissue structures are damaged, microglia undergo several dynamic changes, such as shortening and swelling of the cellular processes, changes in the surface phenotypes and secretory mediators, and increased proliferation response. These changes in cell morphology and function are known as "activated state" [26]. Microglia activation is a common pathological feature of a range of neurodegenerative diseases, including Alzheimer's disease (AD) [27-29]. Also, microglia have a major role in NPSLE, and their abnormal activation has been detected in the hippocampus of several strains of lupus-prone mice (NZB/NZW, MRL/ lpr, and $\mathrm{Fc}_{\mathrm{R}} \mathrm{RIIB}^{-1-} \mathrm{Yaa}$ ) [30]. In the process of continuous inflammation, activated microglia-phagocytosed 
astrocytes promote neuronal apoptosis and aggravate depression index and cognitive dysfunction in mice with lupus [31-33]. Lupus antibodies, DNRAbs, directly activate microglia-mediated neuronal damage and impair cognitive performance [34]. Hence, in this study, we explored the role of microglia in the cognitive dysfunction of NPSLE mice. We analyzed and compared the metabolites in the hippocampal tissues of the validated neuropsychiatric lupus model and control mice. Based on these variations, we found that PKM2 might be the key molecule inducing NPSLE. We then explored SLE-mediated cognition and brain injury pathogenesis.

\section{Materials and methods Materials and reagents}

Flow cytometry antibodies and reagents: FITC-conjugated anti-mouse CD11b, APC-conjugated anti-mouse CD45, PE-conjugated anti-mouse LAMP1 and PE-conjugated anti-mouse CD86 were purchased from Biolegend (USA), anti-CD16/CD32 Fc Block and myelin removal reads were purchased from Miltenyi Biotec (Germany). Western blot and immunofluorescence (IF) antibodies: IBA-1 (R\&D systems, USA); VGLUT1 (Santa Cruz Biotechnology, USA); LAMP1 and CD68 were purchased from Abcam (United Kingdom); albumin, PSD95 and PKM2 were purchased from Proteintech (China). $\beta$-catenin, Cyclin-D1, c-Myc, ENO1, PFKFB3, HK1, LDHA, PDK1, GLUT1 were purchased from Cell Signaling Technology (USA), horseradish peroxidase (HRP)-linked goat anti-rabbit IgG (Fcmacs, China), NeuN (Santa Cruz Biotechnology), donkey anti-rat IgG H\&L (Alexa Fluor ${ }^{\circledR}$ 647) (Abcam), goat anti-mouse IgG-TRITC (Abcam), rabbit anti-goat IgG-FITC (Fcmacs). ELISA kit: Maltose and Glucose assay kit (RayBiotech, USA), L-lactate assay kit (Cayman, USA), interleukin (IL)-6 (Fcmacs, China), IL-1 $\beta$ (Fcmacs, China). TRIzol reagent and SYBR green dye were bought from Invitrogen (Carlsbad, USA). PKM2 overexpression plasmid was purchased from Nanjing Jereh Company (China), and RFectPM Eukaryotic DNA Transfection Kit was purchased from Changzhou EMI Company (China). $\beta$-catenin protein inhibitor (KYA1797K) was purchased from MCE Company (USA).

\section{Adeno-associated virus (AAV) construction}

The AAV used in this study was constructed as previously reported [35]. Briefly, AAV9 carrying the IBA-1 promoter driving the expression of shRNA targeting PKM2 (AAV9-IBA-1 promoter-shPKM2, indicated as AAV9-shPKM2). PKM2 shRNA was produced by Shanghai GeneChem Co., Ltd. (sense: CATGGTCCT GCTGGAGTTCGTG, antisense: CATTCTAGTTGT GGTTTGTCC).

\section{Animals and treatment}

About 6- to 8-week-old female MRL/MpJ-Fas ${ }^{\text {lpr }}$ (MRL/ lpr) and C57BL/6 (control) mice [36] were obtained from Shanghai Slaccas Laboratory Animal Breeding Company (Shanghai, China). All mice were maintained under specific pathogen-free (SPF) conditions at a $12 \mathrm{~h}$ light/ dark cycle and $20-22{ }^{\circ} \mathrm{C}$. The animals were allowed free access to drinking water. The mice were acclimatized to these housing conditions for at least one week. Sixty mice were randomly divided into the following groups: control $(n=20), \quad \mathrm{MRL} / \mathrm{lpr}(n=20)$, Vector (MRL/lpr + AAV9vector, $n=10$ ), shPKM2 (MRL/lpr + AAV9-shPKM2, $n=10$ ). For AAV9 treatment groups, AAV9-shPKM2 or AAV9-vector were stereotactically injected into the lateral ventricle of MRL/lpr mice when MRL/lpr mice grown to 10-week-old. Viruses were diluted with phosphate-buffered saline (PBS), each mouse received an injection of $4 \mu \mathrm{L}(5 \mathrm{E} 10 \mathrm{vg})$ viruses. The intracerebroventricular (ICV) injection were performed perpendicular to the skull ( $x=0.8 \mathrm{~mm}, y=0.2 \mathrm{~mm}, z=2 \mathrm{~mm}$ ) using a microprocessor-controlled mini-pump, delivery was performed at a rate of $500 \mathrm{~nL} / \mathrm{min}$. After injection, the needle was left in place for $5 \mathrm{~min}$ prior to slowly retracting it from the ventricles. Afterward the mice were left underneath a warm light to recover their mobility. When MRL/ lpr mice grown to week 20, the mice were sacrificed [37]. The experiments on mice were approved by Institutional Animal Care and Use Committee, Nanjing University, and all experiments were performed in accordance with relevant guidelines and regulations.

\section{Metabolites extraction}

Hippocampus was dissected on a cold plate and frozen in liquid nitrogen. The tissue was homogenized in $80 \%$ methanol (made with methanol and water) on ice, vortexed for $30 \mathrm{~s}$, sonicated for $10 \mathrm{~min}$ in an ice-water bath, and incubated for $1 \mathrm{~h}$ at $-20{ }^{\circ} \mathrm{C}$ to precipitate the proteins. The supernatant from the remaining sample was obtained by centrifugation at $12,000 \times g$ for $15 \mathrm{~min}$ at $4{ }^{\circ} \mathrm{C}$. The extracts were dried in a vacuum concentrator without heating. A volume of $100 \mu \mathrm{L}$ extraction solvent (V acetonitrile: $\mathrm{V}$ water $=1: 1$ ) was added for reconstitution, which was vortexed $30 \mathrm{~s}$ and sonicated for $10 \mathrm{~min}$ in a $4{ }^{\circ} \mathrm{C}$ water bath, followed by centrifugation for $15 \mathrm{~min}$ at $12,000 \times g$ at $4{ }^{\circ} \mathrm{C}$. The supernatant $(60 \mu \mathrm{L})$ of each sample was analyzed for metabolites [38]. 


\section{Metabolomic analysis by liquid chromatography-mass spectrometry (LC-MS)}

LC-MS/MS analyses were performed using a UHPLC system (1290, Agilent Technologies Santa Clara, CA, USA) with a UPLC HSS T3 column $(2.1 \mathrm{~mm} \times 100 \mathrm{~mm}$, $1.8 \mu \mathrm{m}$ ) coupled to Q Exactive (Orbitrap MS, Thermo, USA). The mobile phase A comprised $0.1 \%$ formic acid in water for normal phase (NP-HPLC) and $5 \mathrm{mmol} / \mathrm{L}$ ammonium acetate in water for reverse phase (RPHPLC). The mobile phase B was acetonitrile. The elution gradient was set as follows: 0 min, $1 \%$ B; 1 min, $1 \%$ B; 8 min, 99\% B; 10 min, 99\% B; 10.1 min, 1\% B; 12 min, $1 \% \mathrm{~B}$. The flow rate was $0.5 \mathrm{~mL} / \mathrm{min}$, and the injection volume was $2 \mu \mathrm{L}$. A QE mass spectrometer was used to record MS/MS spectra in an information-dependent manner during the LC-MS experiment. In this mode, acquisition software (Xcalibur 4.0.27, Thermo) continuously evaluates the full-scan survey MS data as it collects and triggers MS/MS spectra acquisition based on the preselected criteria. The electrospray ionization (ESI) source conditions were set as follows: sheath gas flow rate of 45 Arb, Aux gas flow rate of 15 Arb, capillary temperature of $400{ }^{\circ} \mathrm{C}$, full MS resolution of 70,000 , MS/MS resolution of 17,500 , collision energy of $20 / 40 / 60 \mathrm{eV}$ in normalized collisional energy (NCE) model, and spray voltage of $4.0 \mathrm{kV}$ (positive, POS) or $-3.6 \mathrm{kV}$ (negative, NEG).

The raw data were converted into the mzXML format using ProteoWizard and processed by MAPS software (version 1.0). The preprocessing results generated a data matrix that consisted of retention time, mass-to-charge ratio $(m / z)$, and peak intensity. An in-house tandem mass spectrometry $\left(\mathrm{MS}^{2}\right)$ database was utilized for metabolite identification. The resulting three-dimensional (3D) data involving peak number, sample name, and normalized peak area were entered into SIMCA14.1 software package (V14.1, Sartorius Stedim Data Analytics AB, Umea, Sweden) for principal component analysis (PCA) and orthogonal projections to latent structure-discriminate analysis (OPLS-DA). PCA showed the distribution of the original data. Supervised OPLS-DA was applied to obtain a high level of group separation and identify variables responsible for classification. Sevenfold crossvalidation was used to estimate the robustness and predictive ability of our model. The permutation test further validated the model. A loading plot was constructed on the basis of OPLS-DA and showed the contribution of variables to differences between the two groups. The first principal component of variable importance in projection (VIP) was obtained to refine the analysis. If $P<0.1$ and $V I P>1$, then the variable was defined as a significantly differential metabolite (SDM) between the groups. The SDMs, obtained from LC-MS, were imported into
MetaboAnalyst 4.0 to explore different potential metabolic pathways in the hippocampus between MRL/lpr and control groups. As shown in Fig. 1C, the bubble plots demonstrated the main influential metabolic pathways [39].

\section{Measuring hippocampal glucose and lactate levels}

The hippocampal tissues of different groups of mice were lysed to obtain hippocampal tissue homogenates. According to the manufacturer's instructions, the Maltose and Glucose Assay Kit (RayBiotech, USA) was used to detect the glucose concentration in the hippocampal tissue homogenates, while the L-Lactate Assay Kit (Cayman, USA) was used to determine the concentration of lactic acid in the hippocampal tissue homogenate according to the manufacturer's protocol. The glucose and lactate levels in mouse hippocampus and celebrate cortex were corrected for that of control group.

\section{Flow cytometry analysis of mouse microglia}

The flow cytometry labeling and experimental method of mouse microglia were designed by Bennett et al. [40]. Six mice per group were used to flow cytometry detection. When the mice were killed, one side hippocampus/ cortex was excised on ice immediately, and the tissue dispersed to prepare a single-cell suspension. According to the manufacturer's instructions, the single-cell suspension was mixed with myelin removal beads, then automatic magnetic cell sorter was applied to remove myelin fragments from the single-cell suspension, so that the microglia could be enriched for labeling and detection by flow cytometry [39]. After sorting, single cells were pre-blocked with anti-CD16/CD32 Fc Block for $10 \mathrm{~min}$, then stained on ice for $30 \mathrm{~min}$ with CD11b-FITC, CD45-APC and CD86-PE, CD86-PE or LAMP1-PE, rinsed twice with PBS, and finally resuspended in $200 \mu \mathrm{L}$ buffer for subsequent evaluation by flow cytometry (BD Accuri $^{\text {TM }}$ C6, BD Biosciences, USA). Data were analyzed using FlowJo software. Microglia were defined as $\mathrm{CD} 11 \mathrm{~b}^{+} \mathrm{CD} 45^{\text {lo }}$, proinflammatory microglia were defined as $\mathrm{CD} 11 \mathrm{~b}^{+} \mathrm{CD} 45^{\mathrm{lo}} \mathrm{CD} 86^{+}$.

\section{Brain pathological evaluation}

Four mice per group were used for pathological evaluation. The whole-brain of the mice was fixed with $4 \%$ polyoxymethylene (PFA) and sliced into 5-mm-thick sections for hematoxylin/eosin (H\&E) staining.

\section{PKM2 detection in microglia and neurons} by immunofluorescence (IF) staining

Double IF staining was carried out to detect the expression of PKM2 in microglia and neurons in hippocampus tissue. Briefly, $8-\mu \mathrm{m}$-thick frozen brain slices were fixed 

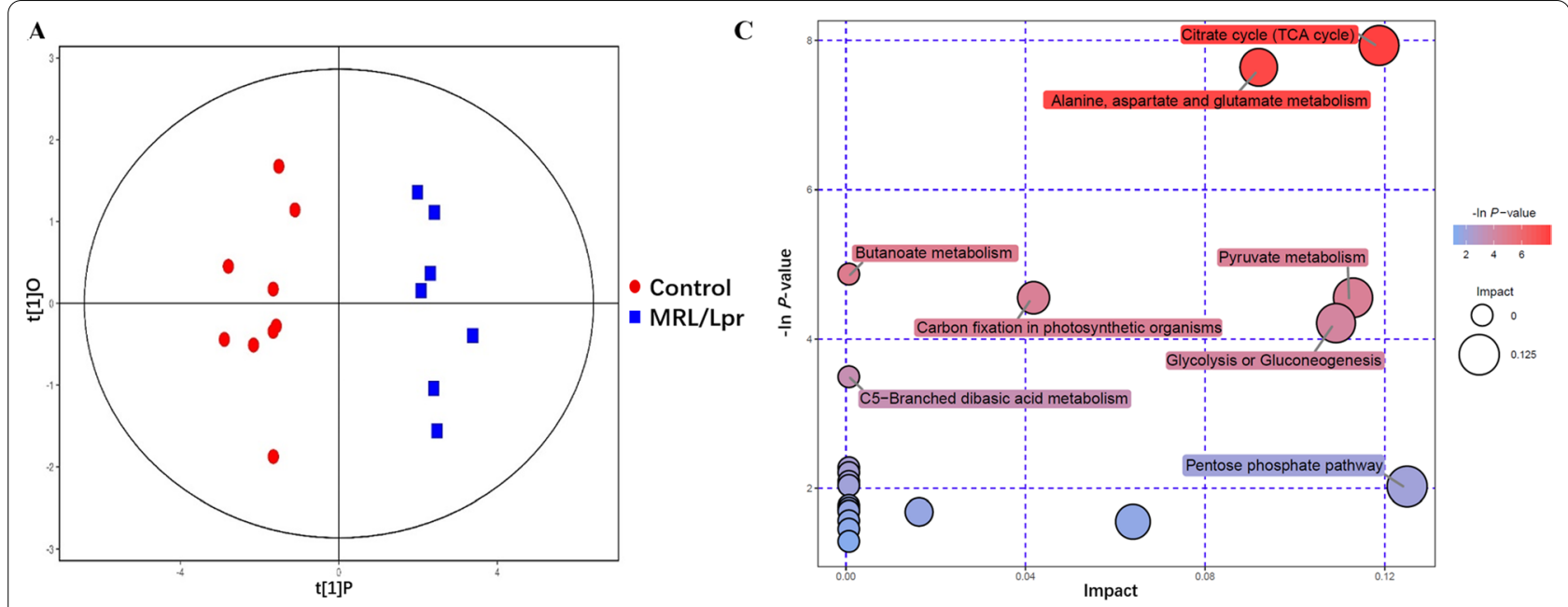

B
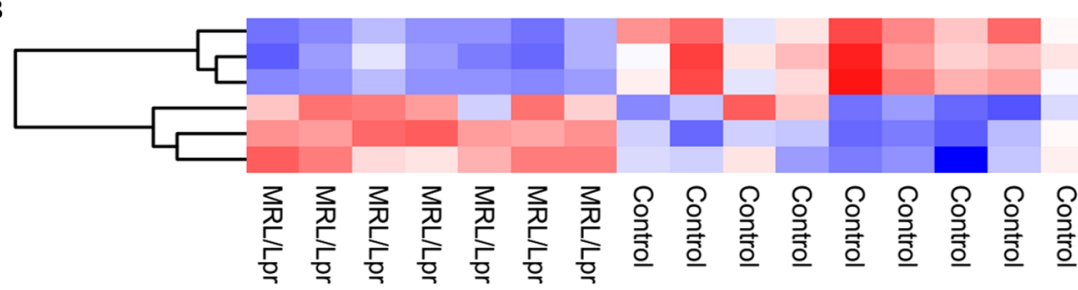

Sedoheptulose 7-phosphate Fructose 6-phosphate Glucose 6-phosphate

Pyruvic acid

L-Lactic acid

Oxoglutaric acid

cid

the

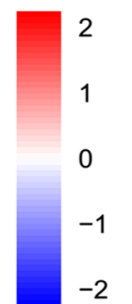

Fig. 1 Central carbon metabolome analysis showing elevated metabolites in glycolysis in MRL/lpr mice hippocampus. A PCA model score scatter plot and OPLS-DA model showing separation of glycolysis metabolites in the hippocampus of control mice (blue) and MLR/lpr group mice (red). B Hierarchical clustering analysis for the significantly differential metabolites of LC-MS between the control and MLR/lpr groups of mice. The relative metabolite level was depicted according to the color scale: red indicates upregulation, whereas blue indicates downregulation. C Metabolomic map of significant metabolic pathways characterized in the hippocampus of control and MLR/lpr groups. The significantly altered pathways based on enrichment and topology analysis are shown in this metabolomic map. The $X$-axis represents pathway impact; $Y$-axis represents pathway enrichment. Large size and dark colors represent the major pathway enrichment and high pathway impact values, respectively

in cold methanol/acetone $(1: 1)$ for $10 \mathrm{~min}$ at $-20{ }^{\circ} \mathrm{C}$. After PBS washes, the samples were blocked with $3 \%$ bovine serum albumin (BSA) in PBS for $60 \mathrm{~min}$ at room temperature, followed by incubation with anti-IBA-1, anti-PKM2, and anti-NeuN primary antibodies (1:200) overnight at $4{ }^{\circ} \mathrm{C}$. Subsequently, the samples were incubated with goat anti-mouse IgG-TRITC and rabbit antigoat IgG-FITC secondary antibodies (1:400) for $1 \mathrm{~h}$ at room temperature in the dark, followed by nuclei staining with 2-(4-amidinophenyl)-1H-indole-6-carboxamidine (DAPI).

To evaluate the neuronal degeneration, double staining of NeuN and the TUNEL was performed using the In Situ Cell Death Detection Kit (Roche Diagnostics) [41]. Briefly, double staining of NeuN and the TUNEL was performed using the In Situ Cell Death Detection Kit (Roche Diagnostics). After immunohistochemical staining of IBA-1 as described above, the sections were washed three times in PBS and then incubated with the TUNEL solution containing FITC-dUTP for $60 \mathrm{~min}$ at $37{ }^{\circ} \mathrm{C}$. Finally, the sections were washed in PBS and mounted for the analysis. All sections were examined by a Nikon Eclipse Ti-U fluorescence confocal microscope, which equipped with a digital camera (FV300, Olympus, Japan).

\section{RNA extraction and quantitative real-time PCR (qPCR)}

Six mice per group were used to assess the expression of mRNA. Total RNA was extracted from cells or tissues using TRIzol reagent. According to the manufacturer's instructions, $1 \mu \mathrm{g}$ total RNA was reverse transcribed in a $20-\mu \mathrm{L}$ reaction system. The oligonucleotide primers used for PCR amplification are listed in Table 1 . All reactions were carried out in triplicate. The expression levels of the target genes were normalized to that of GAPDH.

\section{Western blot}

Proteins of one side hippocampus/cortex were extracted by standard techniques [42]. Four mice per group were used for Western blot detection. Typically, total proteins 
Table 1 Primers used for q-PCR in this study

\begin{tabular}{lll}
\hline Gene & Forward & Reverse \\
\hline GAPDH & GCATGGCCTTCCGTGTTCC & GGGTGGTCCAGGGTTTCTTACTC \\
PKM2 & TCAGAGCTCCAACGCTTGTAGAACTCACTC & CCGCTCGAGAAATGGAAGGTGGAGGG \\
ENO1 & GCCTCCTGCTCAAAGTCAAC & AACGATAGACACCATGACG \\
HK1 & TGCCATGCGGCTCTCTGATG & GCCACAACTGTAGGGTCGT \\
PFKFB3 & ATTGCGGTTTCGATGCCAC & GCAACTTGCAGTTCGGGC \\
LDHA & ATGGCAACTCTAAAGGATCA & CCCATGCATTTGTGCCTACC \\
PDK1 & AGGCAAAGGAAGTCATCTCA & GAACACCTGGGCGATGAGGATG \\
\hline
\end{tabular}

were separated by sodium dodecyl sulfate-polyacrylamide gel electrophoresis (SDS-PAGE), then the total protein were transferred to polyvinylidene fluoride (PVDF) membranes (Millipore Co, Bedford, MA, USA). The membranes were blocked in 5\% BSA dissolved in TBST (50 mM Tris/HCL, pH 7.6, $150 \mathrm{mM} \mathrm{NaCl}$ and $0.1 \%$ Tween-20) for $2 \mathrm{~h}$ at room temperature and probed with indicated primary antibodies overnight at $4{ }^{\circ} \mathrm{C}$, washed the membrane three times with TBST, followed by incubation with appropriate HRP-linked secondary antibody $2 \mathrm{~h}$ at room temperature, washed the membrane three times. The immunoreactive bands were visualized using enhanced chemiluminescence (ECL) plus Western blot detection reagents (Supersignal ${ }^{\mathrm{TM}}$ West Pico PLUS, Thermo, USA).

For the membranes that needs to be stripped of antibodies, the membranes were washed with TBS three times, incubated with restore Western blot stripping buffer (Thermo scientific, USA) in the dark at $37^{\circ} \mathrm{C}$ for 20 min, washed with TBS 3 times [43, 44]. Then, the membranes were re-blocked in 5\% BSA for 30-60 min at room temperature, and probed with indicated primary antibodies overnight at $4{ }^{\circ} \mathrm{C}$, subsequent steps are the same as conventional WB. The gray values were analyzed by Image J software (National Institutes of Health, Bethesda, MD, USA).

\section{Cytokine detection by enzyme-linked immunosorbent assay (ELISA)}

Four mice per group were used for ELISA detection. ELISA was used to detect the content of inflammatory factors (IL-1 $\beta$ and IL-6) in the hippocampus of mice using the commercial kits, according to the manufacturer's instructions. The mouse hippocampal tissues protein extract was at dilution of 1:5.

\section{Cell culture and treatment}

Microglia cell line BV2 cells and neuronal cell line HT22 cells were generous gifts from Prof. Tianjiao Xia, Nanjing
University and cultured in Dulbecco's modified eagle's medium (DMEM) containing 10\% fetal bovine serum (FBS; Gibco) at $37{ }^{\circ} \mathrm{C}$ with $5 \% \mathrm{CO}_{2} .2 \times 10^{5} \mathrm{BV} 2$ cells/ $\mathrm{mL}$ was seeded in 6-well, when cell confluency exceeded $30 \%$, the cells were transfected with PKM2 overexpression plasmid. The cells were treated with $\beta$-catenin inhibitor (KYA1797K), at a $200 \mathrm{ng} / \mathrm{mL}$ concentration. After that, BV2 cells were co-cultivated with HT22 cells at a ratio of $1: 3$ for $24 \mathrm{~h}$ and collected cells for subsequent experiments.

\section{Detection of microglial phagocytosis}

The phagocytic capacity of microglia was determined using the method described previously [45]. Briefly, BV2 cells were seeded into 6-well plates at a density of $2 \times 10^{5}$ cells/well in DMEM medium. The fluorescent yellow-green labeled 1- $\mu \mathrm{m}$ amine-modified polystyrene latex beads were mixed with FBS at a ratio of $1: 5$ and incubated in a $37^{\circ} \mathrm{C}$ water bath for $1 \mathrm{~h}$, then this mixture was diluted with DMEM complete medium to achieve the final concentration of fluorescent beads and FBS in DMEM as $0.01 \%(\mathrm{v} / \mathrm{v})$ and $0.05 \%(\mathrm{v} / \mathrm{v})$, respectively. Subsequently, the cells were washed twice with PBS and incubated with DMEM, containing fluorescent beads, for $1 \mathrm{~h}$ at $37^{\circ} \mathrm{C}$. Finally, the cells were collected for flow cytometry detection.

\section{Behavioral assays}

Ten mice per group were used to carry out behavioral detection. The majority of the 16-week-old MRL/lpr mice exhibited characteristic cognitive dysfunctions [46]. Morris water maze (MWM) test was conducted to assess hippocampus-dependent spatial learning and memory functions in rodents $[47,48]$. The MWM tests included two parts: the spatial acquisition and probe trials. The spatial acquisition trial was performed for five consecutive days. In each trial, the mouse was allowed to search the platform within $60 \mathrm{~s}$, and could stay on the platform for $5 \mathrm{~s}$ after it was located. The mouse that failed to 
find the platform in $60 \mathrm{~s}$ was guided to it manually and ordered to remain on the platform for $15 \mathrm{~s}$, which was regarded as latency. The time spent on searching and mounting the platform (latency) was calculated. On day 6 , a probe trial was performed for reference memory by removing the platform. The mice were randomly placed into two selected quadrants, which had different distances to the platform and were allowed to swim freely for $60 \mathrm{~s}$. The percentage of time spent in the target quadrant and platform crossings was recorded, analyzed, and considered as an indicator of memory retention.

The fear-conditioning paradigm was also assessed [49, 50]. Briefly, mice were trained to associate cage context or an audiovisual cue with an aversive stimulus (footshock). The test was administered over 2 days. On day 1 , mice were placed in a cage and exposed to two periods of $30 \mathrm{~s}$ each of paired cue light and $3000-\mathrm{Hz}$ tone, followed by a 2 -s foot-shock $(0.8 \mathrm{~mA})$ with a 180 -s interval. On day 2 , mice were subjected to two trials. In the first trial assessing contextual memory, mice were re-exposed to the same cage context, and freezing behavior was measured over 1-3 min, using a FreezeScan tracking system (CleverSys, Inc., Reston, VA). In the second trial measuring cued memory, mice were placed in a novel context and exposed to the same cue light and tone from day 1 after 2 min of exploration. The freezing behavior was measured for 1-3 min following the cue.

\section{Statistical analysis}

All data are presented as mean \pm standard error of mean (SEM), and each experiment included triplicate sets. The significant differences among groups with one independent variable were determined by one-way analysis of variance (ANOVA) with a Tukey's multiple comparisons test for planned comparisons. $P$-value $\leq 0.05$ was considered significant. GraphPad Prism 5 was used for data analysis (GraphPad Software Inc., CA, USA).

\section{Results}

Central carbon metabolome analysis reveals that elevated glycolysis is involved in hippocampal tissues in MRL/lpr lupus mice

The hippocampus has an important part in the pathogenesis of NPSLE [51, 52]. To understand the alterations on central carbon metabolism in the hippocampus of neuropsychiatric lupus mice, the hippocampus from 20-week-old female MRL/lpr and control mice was harvested for targeting metabolite analysis. Eleven metabolites, including 6-phosphogluconic acid, were differentially expressed in the two groups (Table 2).

Based on PCA and OPLS-DA analysis, a significant difference in the metabolites of the hippocampus were observed between MRL/lpr and control mice (Fig. 1A, B Additional file 1: S1A, S1B), and all the samples were within the $95 \%$ confidence interval (Hotelling's T-squared ellipse). Briefly, the levels of pyruvic acid, L-lactic acid, and oxoglutaric acid $(P \leq 0.05)$ were significantly elevated in the hippocampus of MRL/lpr mice, while sedoheptulose 7-phosphate, fructose 6-phosphate, and glucose 6 -phosphate $(P \leq 0.05)$ were significantly decreased (Fig. 1B). In addition, signaling pathway analysis revealed elevated glycolysis and pyruvate in hippocampal tissues in MRL/lpr mice (Fig. 1C), indicating that the glycolysis metabolic pathway in the hippocampus of lupus mice was significantly altered.

\section{NPSLE induces increases in glucose consumption and lactic} acid production in the hippocampus

Based on the metabolic profiles in the lupus hippocampus, we further detected glucose consumption and the production of lactic acid in the hippocampal tissues via

Table 2 Metabolic overall analysis among the NPSLE model group and control group

\begin{tabular}{lllll}
\hline Metabolite name & Log-fold change & P-value & VIP & Q-value \\
\hline 6-Phosphogluconic acid & $6.176 \mathrm{E}-03$ & 0.986 & 0.346 & 0.329 \\
D-Glucose & $9.690 \mathrm{E}-02$ & 0.176 & 0.528 & 0.080 \\
D-Ribulose-5-phosphate & $4.066 \mathrm{E}-01$ & 0.03 & 1 & 0.017 \\
Fructose 6-phosphate & $-1.717 \mathrm{E}+00$ & $4.312 \mathrm{E}-04$ & 1.325 & $5.190 \mathrm{E}-04$ \\
Glucose 6-phosphate & $-2.832 \mathrm{E}+00$ & 0.001 & 1.378 & $6.017 \mathrm{E}-04$ \\
L-Lactic acid & $3.554 \mathrm{E}-01$ & $9.050 \mathrm{E}-06$ & 1.474 & $3.301 \mathrm{E}-05$ \\
Malic acid & $6.597 \mathrm{E}-03$ & 0.964 & 0.148 & 0.324 \\
Oxoglutaric acid & $3.296 \mathrm{E}-01$ & 0.026 & 0.829 & 0.016 \\
Pyruvic acid & $7.167 \mathrm{E}-01$ & 0.038 & 0.855 & 0.020 \\
Sedoheptulose 7-phosphate & $-1.841 \mathrm{E}+00$ & $1.881 \mathrm{E}-04$ & 1.414 & $3.430 \mathrm{E}-04$ \\
Succinic acid & $-2.718 \mathrm{E}-01$ & 0.261 & 0.580 & 0.115 \\
\hline
\end{tabular}




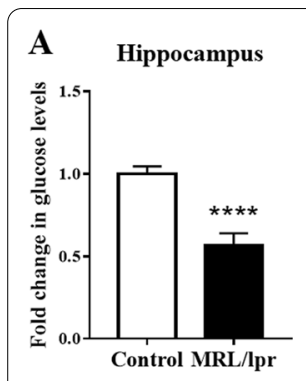

C

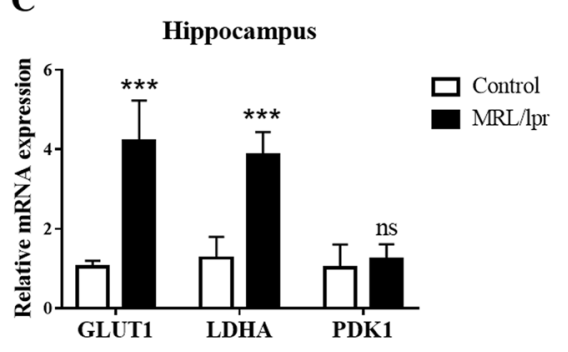

$\mathbf{E}$

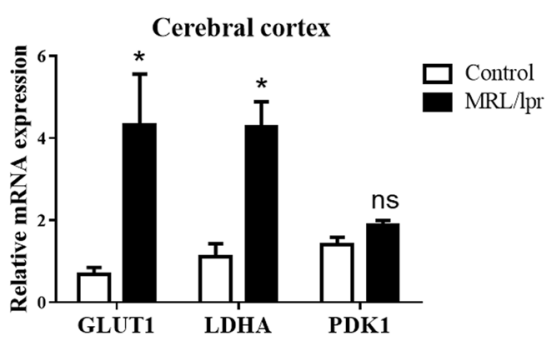

D

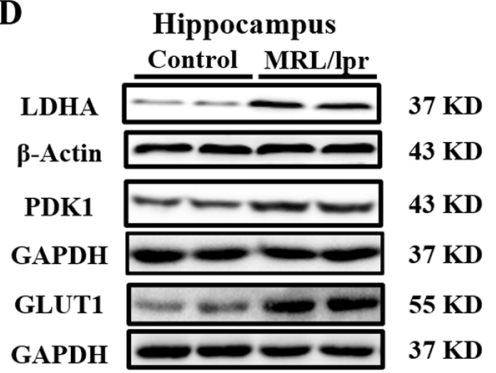

$\mathbf{F}$

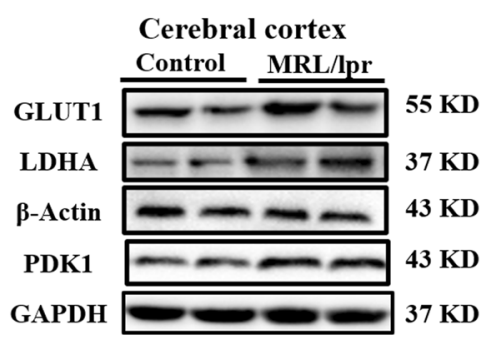

B
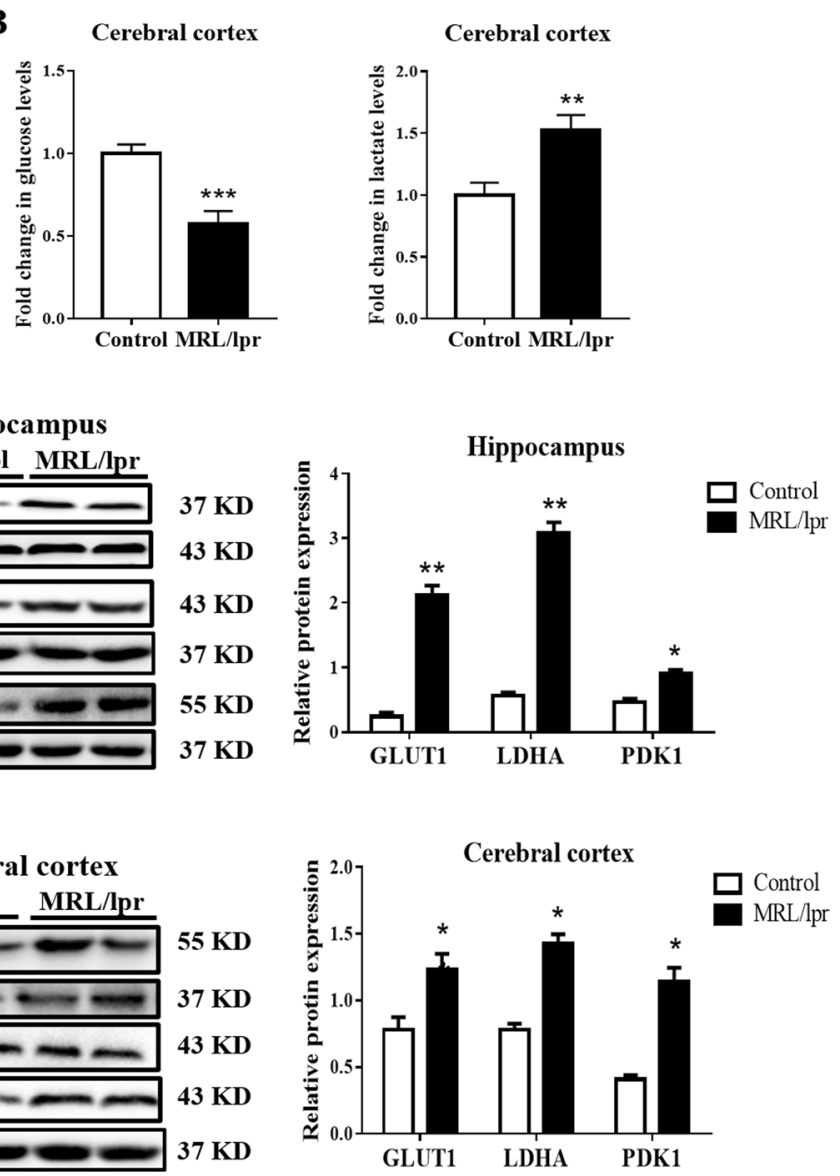

Fig. 2 NPSLE promotes cell glycolysis in the hippocampus, with increased expression of GLUT1, PDK1, and LDHA. A Glucose levels and lactate production in the hippocampus of the MLR/Ipr mice were normalized to that of the control group mice, $n=6$. $\mathbf{B}$ Glucose levels and lactate production in the cerebral cortex of the MLR/Ipr mice were normalized to that of the control group mice, $n=6$. C The mRNA expression of GLUT1, LDHA, and PDK1 in the hippocampus of control and MLR/Ipr groups, $n=6$. D Western blot quantification of GLUT1, LDHA, and PDK1 in the hippocampus of control and MLR/lpr groups, $n=4$. E The mRNA expression of GLUT1, LDHA, and PDK1 in the cerebral cortex of the control and MLR/lpr groups, $n=6$. F Western blot quantification of GLUT1, LDHA, and PDK1 in the cerebral cortex of control and MLR/lpr groups, $n=4$. Data are presented as mean scores $\pm \mathrm{SEM},{ }^{*} P \leq 0.05,{ }^{* *} P \leq 0.01,{ }^{* *} P \leq 0.001$

ELISA. Compared to the control group, the glucose consumption in the NPSLE group significantly increased (about twofold decreased, $P \leq 0.005$, Fig. 2A), accompanied with the increase lactate production (about 1.5-fold increased, $P \leq 0.01$, Fig. $2 \mathrm{~A}$ ); the same was also observed in the cortical tissues (Fig. 2B). Furthermore, we built another murine model of lupus induced by imiquimod (IMQ) [53]. In the hippocampus and cerebral cortex of the IMQ group, the glucose consumption and lactate production were significantly increased, which was consistent with the results from the typical NPSLE mice model ( $P \leq 0.01$, Additional file 1: Fig. S2A). Notably, glucose was transported across the plasma membrane by glucose transporters (GLUT); the mRNA and protein expression of GLUT1 (4.2-fold and sixfold increase, respectively, $P \leq 0.05)$ were significantly upregulated in the hippocampus of lupus mice compared to control mice (Additional file 1: Fig. S2B-D). The mRNA and protein levels of hippocampal lactic dehydrogenase kinase A (LDHA) and pyruvate dehydrogenase kinase 1 (PDK1) (sixfold and twofold increase, respectively, $P \leq 0.05$ ) were also upregulated (Fig. 2C, D). Cortical GLUT1, LDHA, and PDK1 levels were upregulated, except for the PDK1 gene $(P \leq 0.05$, Fig. $2 \mathrm{E}, \mathrm{F}$ and Additional file 1 : Fig. $\mathrm{S} 2 \mathrm{C})$.

To sum up, these results indicated that the glycolysis was altered in the hippocampus of NPSLE mice, showing increased glucose consumption and lactic acid production.

\section{Glycolysis promotes PKM2 activation in hippocampal microglia}

Based on the glycolysis pathway (Fig. 3A), we detected the expression of several critical glycolytic enzymes (HK1, PFKFB3, ENO1, and PKM2) in the hippocampus. 


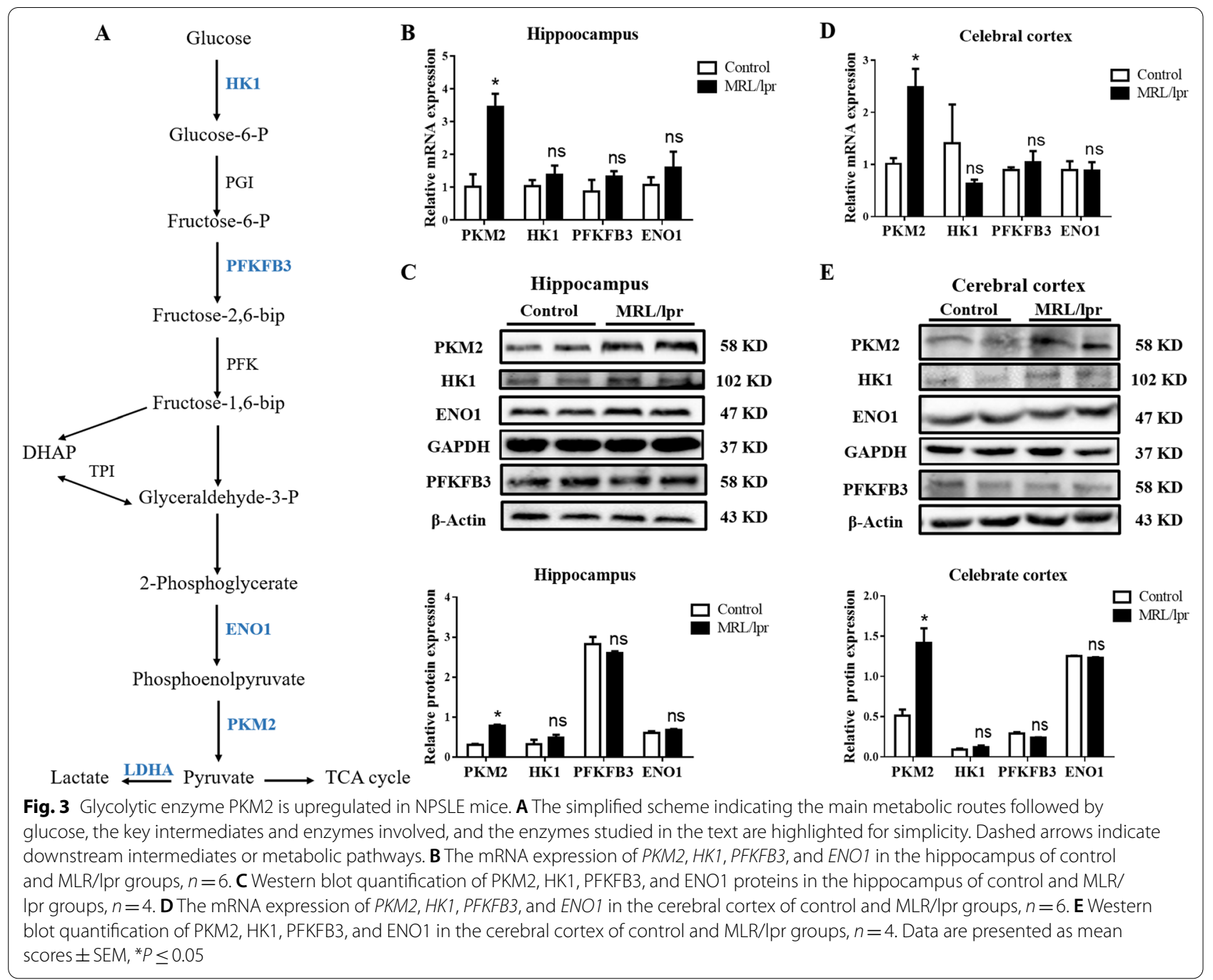

Interestingly, only PKM2 expression was significantly increased (3.2-fold increase, $P \leq 0.05$ ), while the other key enzymes remained unchanged (Fig. 3B, C, and Additional file 1: Fig. S3A); similar results were observed in cortical tissue (Fig. 3D, E and Additional file 1: Fig. S3B). These data suggested that the abnormal metabolism of glycolysis in the brain of NPSLE mice could be attributed to the upregulation of PKM2.

To further explore in which cell types PKM2 was expressed, we analyzed the colocalization of PKM2 with microglia $\left(\mathrm{IBA}-1^{+}\right)$and neuron $\left(\mathrm{NeuN}^{+}\right)$by IF. Results showed that the colocalization of PKM2 with microglia in the hippocampus of MRL/lpr mice was significantly increased (mean $531 \pm 43$ cells $/ \mathrm{mm}^{2}$ ) compared to the control mice (mean $233 \pm 52$ cells $/ \mathrm{mm}^{2}, P \leq 0.01$, Fig. 4A, $B)$, which indicated that the abnormal glycolysis might be related to the upregulation of PKM2 in microglia.

As one of the key enzymes of glycolysis, PKM2 has a dimeric and a tetrameric form; however, the biological effects between the tetramer and the dimer were significantly different [54-57]. Our results showed that tetrameric formation of PKM2 (232 kDa) was enhanced in the hippocampus of MRL/lpr mice compared to control mice (Additional file 1: Fig. S4A), which was consistent with the results of glucose consumption in the hippocampus of MRL/lpr mice. Simultaneously, we observed that the level of PKM2 dimer $(116 \mathrm{kDa})$ in the hippocampus of MRL/lpr mice was increased (Additional file 1: Fig. S4B), which suggested that the dimer PKM2 might enter the nuclear to regulate nuclear transcription.

In order to verify this inference, we stimulated BV2 microglia with Toll-like receptor7 (TLR7) agonist R848 in vitro to simulate the lupus environment. After R848 induction, total PKM2 in BV2 cells were over-expressed. Meanwhile, cytoplasmic PKM2 decreased, which suggested that PKM2 enters the nucleus more under R848 stimulation (Additional file 1: Fig. S4C). Similarly, the immunofluorescence staining intuitively confirmed that 


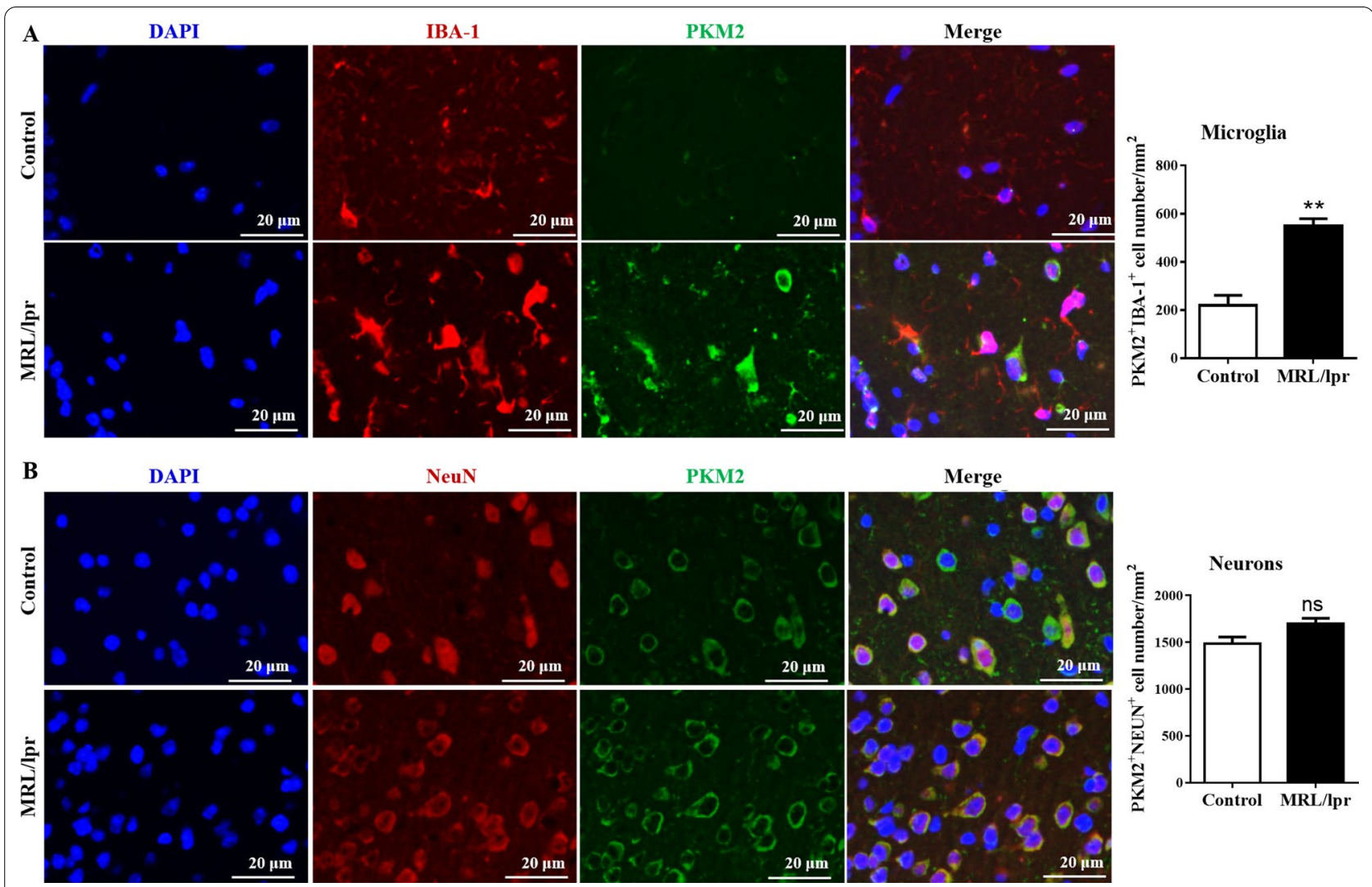

Fig. 4 The colocalization of PKM2 with microglia increases in the hippocampus of MRL/Ipr mice. A The expression levels of IBA-1 (red) and PKM2 (green) and their colocalization (yellow) in the hippocampus of control and MLR/Ipr groups mice were detected by IF staining; Bar $=20 \mu \mathrm{m}, n=4$. B The expression levels of NEUN (red) and PKM2 (green) and their colocalization (yellow) in the hippocampus of control and MLR/lpr groups mice detected by IF staining; $\operatorname{Bar}=20 \mu \mathrm{m}, n=4$. Data represent the mean scores \pm SEM, ${ }^{* *} P \leq 0.01$

more PKM2 entered the nucleus after R848 treatment (Additional file 1: Fig. S4D).

These data indicated that the increased expression of dimer and tetramer PKM2 in the hippocampus of MRL/ lpr mice might be involved in the occurrence and development of NPSLE.

\section{Increased microglial engulfment of neuronal synapses in the hippocampus of NPSLE mice}

To understand how microglia respond to the pathological PKM2 in the hippocampus of lupus mice, we calculated the percentage and phenotype of microglia in the hippocampus of two group mice by flow cytometry; the experimental procedure is shown in Additional file 1: Fig. S5A and B. Results showed that the microglia population $\left(\mathrm{CD} 45^{\text {low }} \mathrm{CD} 11 \mathrm{~b}^{+}\right)$in MRL/lpr mice was significantly higher than that of control mice $(16.1 \pm 1.63 \%$ vs. $7.84 \pm 0.23 \%, P \leq 0.001$, Fig. 5A). Besides, the expression of CD86 on microglia was upregulated in MRL/ lpr lupus mice (median fluorescence intensity (MFI): $2062.87 \pm 45.39$ vs. $1858.37 \pm 50.24, P \leq 0.001$, Fig. $5 \mathrm{~B}$ ). Apart from this, immunoblotting showed that the expression of IBA-1 and CD68 was obviously increased in the hippocampus of NPSLE mice compared to the control mice (Fig. 5D). In addition, the IF images of hippocampus confirmed that the population of IBA- $1^{+}$ microglia cell was significantly increased (mean 28 cells/ $\mathrm{mm}^{2}$ [CI 200-300 cells $/ \mathrm{mm}^{2}$ ] vs. 716 cells $/ \mathrm{mm}^{2}$ [CI 600800 cells $/ \mathrm{mm}^{2}$ ] controls; $P \leq 0.01$ ), with microglial thick cell bodies and abundant branches, and the expression of CD68 on IBA- $1^{+}$microglia was also elevated (Fig. 5E). Also, in the IMQ-induced lupus mouse model, microglial activation in the hippocampus increased markedly (Additional file 1: Fig. S6A, B, D, E). All these results suggested that hippocampus microglia were activated in lupus mice.

Abnormal phagocytosis of microglia has a major role in CNS and impairs cognitive functions such as learning and memory [58-60]. The expression of lysosome-associated membrane protein 1 (LAMP1) in the hippocampus was evidently upregulated, as shown by the Western blot results $(P \leq 0.001$, Fig. $5 \mathrm{D}$ and Additional file 1: Fig. S6C). Meanwhile, flow cytometry detection showed that microglial LAMP1 also 


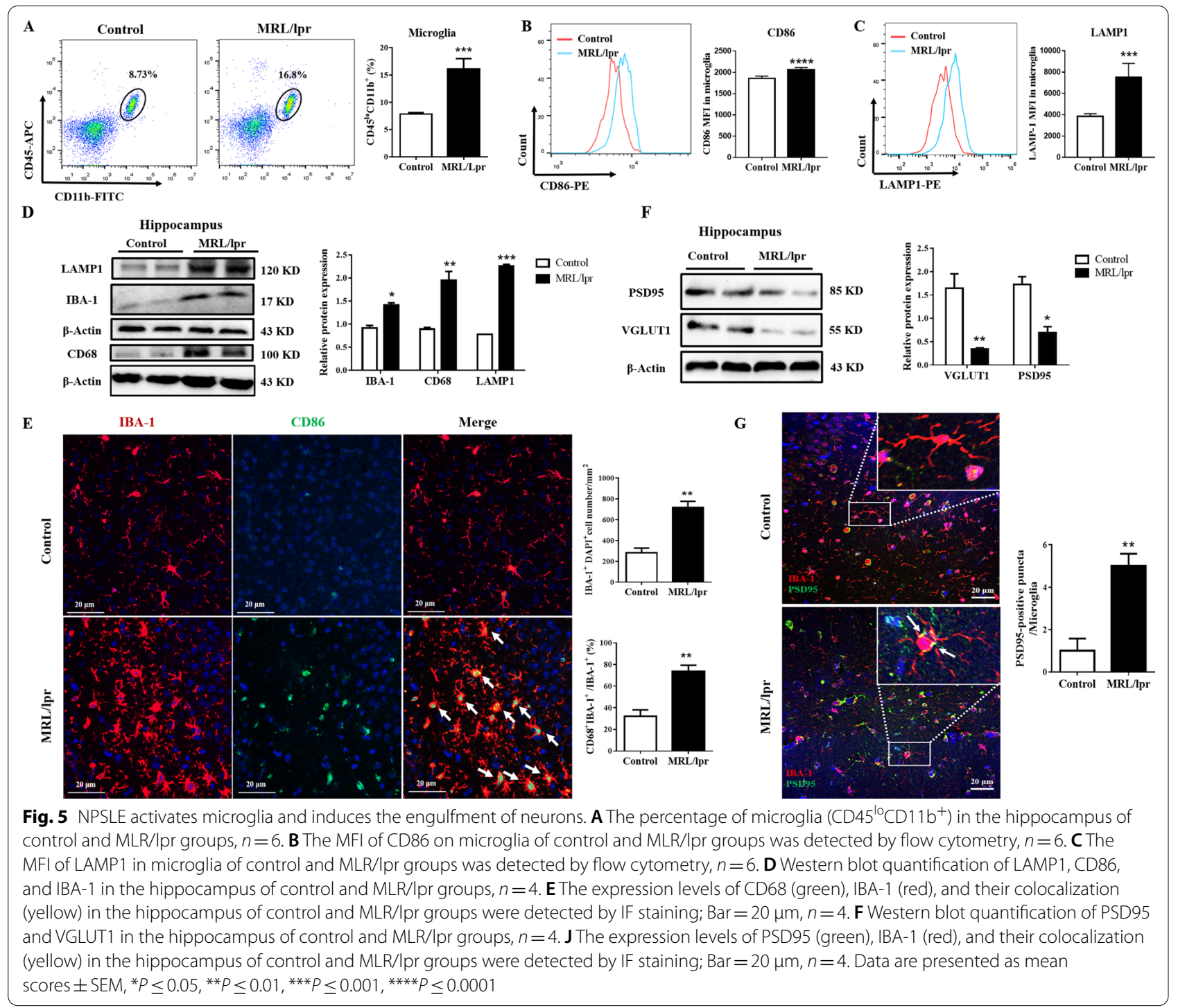

increased (MFI: $7499.2 \pm 1176.38$ vs. $1860.8 \pm 210$ controls, $P \leq 0.001$, Fig. 5 C). All above results indicated that the phagocytic function of hippocampal microglia in NPSLE mice was altered.

To further confirm our points, we detected VGLUT1 and PSD95 (neuronal synaptic markers) in the murine hippocampus. Compared to control mice, the expression of PSD95 (sevenfold decrease, $p \leq 0.05$ ) and VGLUT1 (2.3-fold decrease, $P \leq 0.05$ ) decreased in NPSLE mice (Fig. 5F), while the colocalization of PSD95 and IBA-1 increased in MRL/lpr mice (Fig. 5G). After stimulation with R848, the phagocytosis of BV2 cells was monitored to assess microglia-mediated synapse engulfment. The results showed that the phagocytosis of BV2 cells increased after R848 stimulation (Additional file 1: Fig. S7A-D). Taken together, it could be deduced that the activation of microglia enhanced in the hippocampus of lupus mice promoted the purge of synapses by microglia.

\section{PKM2 facilitates microglial engulfment of neurons via $\beta$-catenin signaling}

In vitro experiments showed that the increase in phagocytosis of BV2 cells was accompanied by a dose- and time-dependent increase in PKM2 after R848 stimulation (Additional file 1: Fig. S7F, G). In order to elucidate the correlation between PKM2 and microglial engulfment of neuronal structures, PKM2-overexpressing plasmids were transfected in BV2 microglia $(P \leq 0.05$, Fig. 6A, B). Next, flow cytometry and Western blot experiments showed that the protein level of LAMP1 was significantly increased after PKM2 overexpression in BV2 cells $(P \leq 0.01$, Fig. $6 \mathrm{C}, \mathrm{D})$. Fluorescent microsphere 

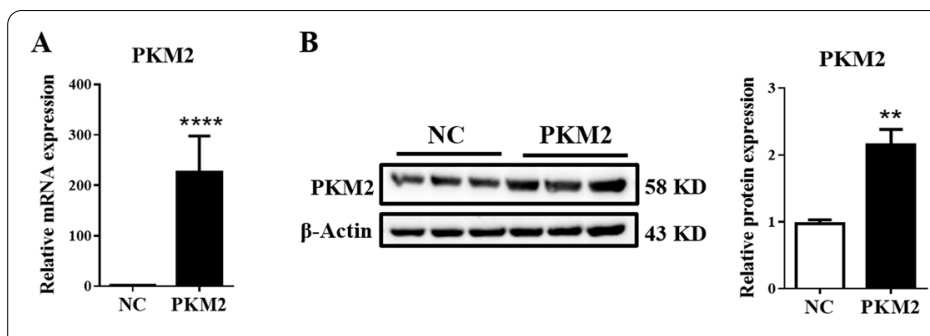

C

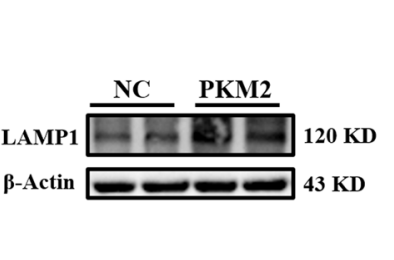

D

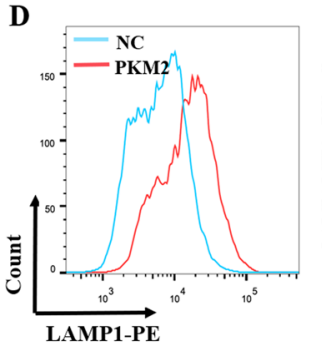

LAMP1-PE

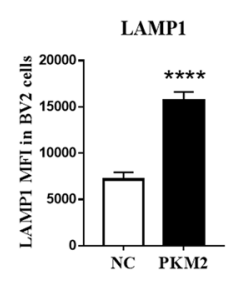

F

E
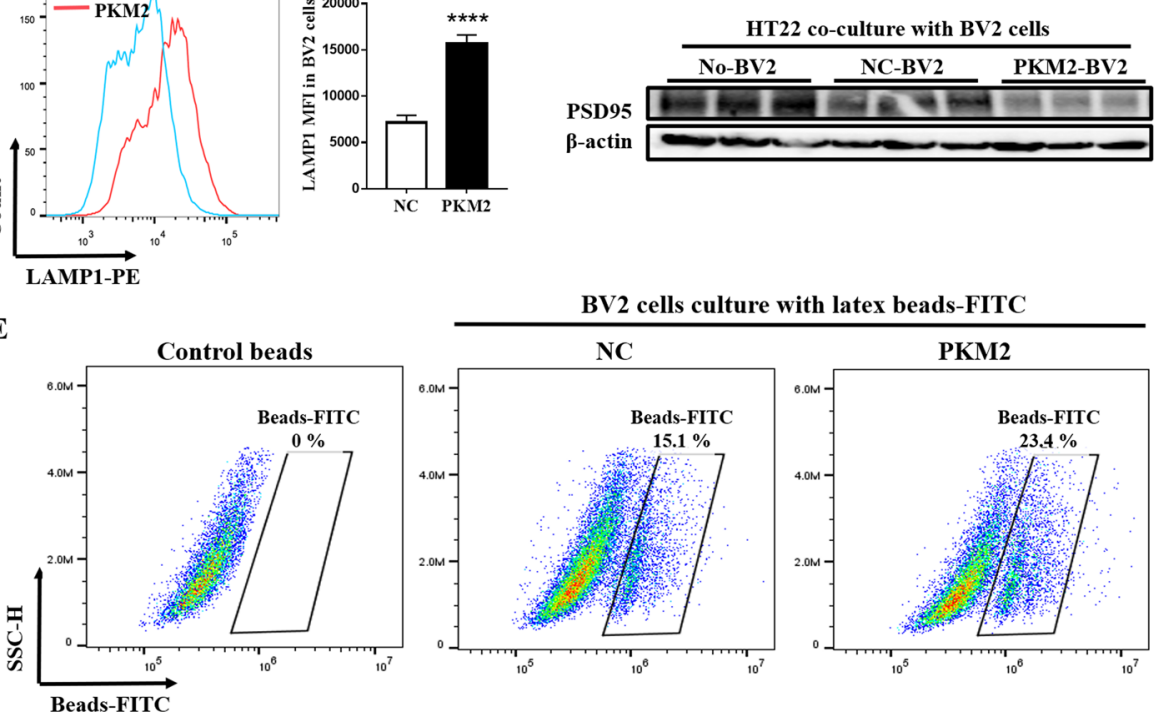
swo $43 \mathrm{KD}$

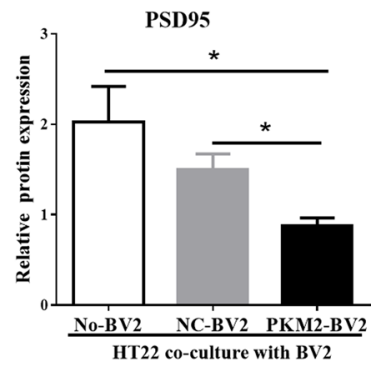

BV2 cells culture with latex beads-FITC
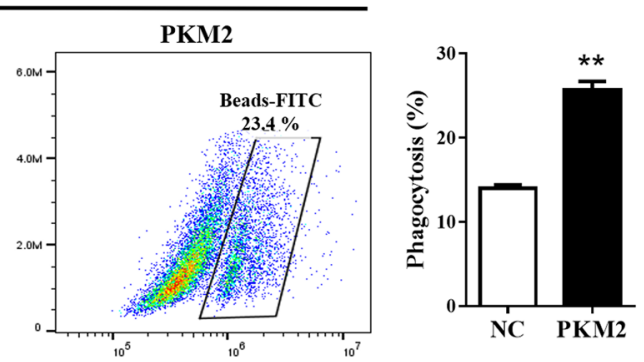

Fig. 6 PKM2 overexpression stimulates phagocytic activity and engulfment of neurons. Transient transfection of BV2 cells with the negative control plasmid (NC) and PKM2 over-expressed plasmid (PKM2); 24 h later, the cells were analyzed for RT-PCR, Western blot, and flow cytometry. A The mRNA expression of PKM2 was detected by RT-PCR. B Western blot quantification of PKM2 on BV2 cells. C Western blot quantification of LAMP1 on BV2 cells. D The expression level of LAMP1 detected by flow cytometry. E After BV2 cells were transfected with NC and PKM2 high expression plasmids, a phagocytic function test was performed, and the phagocytosis of BV2 cells were detected by flow cytometry. $\mathbf{F}$ After transfected with NC and PKM2 high expression plasmids, BV2 cells were co-cultured with HT22 cells, then Western blot was used to detect the protein level of PSD95. Data represent the mean scores \pm SEM, ${ }^{*} P \leq 0.05,{ }^{* *} P \leq 0.01,{ }^{* * *} P \leq 0.0001 . n=3$ per group

experiments proved that the phagocytosis of BV2 cells was significantly enhanced after overexpression of PKM2 (25.45 $\pm 0.81 \%$ vs. $13.57 \pm 0.74 \%$ control; $P \leq 0.01$, Fig. 6E). Moreover, a co-culture of BV2 and HT22 cells indicated that the expression of PSD95 on HT22 was downregulated in BV2 cells overexpressing PKM2 (Fig. 6F).

The $\beta$-catenin signaling pathway is involved in the development of various CNS diseases [61-63]. Hence, we speculated that PKM2 upregulates microglial phagocytosis through the $\beta$-catenin signaling pathway. We detected the expression of the downstream molecular of the $\beta$-catenin signaling pathway on BV2 cells after PKM2 overexpression to confirm this speculation. Results showed that $\beta$-catenin and the downstream target genes/ proteins c-Myc and Cyclin-D1 were upregulated after overexpression of PKM2 $(P \leq 0.05$, Fig. 7A, B). In addition, these molecules also significantly increased after
R848 stimulation $(P \leq 0.05$, Additional file 1: Fig. S8A-D), and the mRNA levels of these genes showed a significant positive correlation with PKM2 (Additional file 1: Fig. S8A, B). Similarly, in vivo, the levels of $\beta$-catenin, c-Myc, and Cyclin-D1 were elevated in the hippocampus of NPSLE mice $(P \leq 0.01$, Fig. $7 C)$. These findings indicated that PKM2 could activate the $\beta$-catenin signaling pathway in vivo and in vitro.

To further demonstrate the involvement of the $\beta$-catenin signaling pathway in neuron and synapse loss in NPSLE mice, we selected KYA1797K to inhibit the expression of $\beta$-catenin [64]. The safe dose of KYA1797K was determined by the CCK 8 experiment, and the final concentration of KYA1797K was selected to be $200 \mathrm{ng} / \mathrm{mL}$ for subsequent experiments (Fig. 7D). Then, fluorescent bead experiments showed that the enhanced microglial phagocytosis post-PKM2 

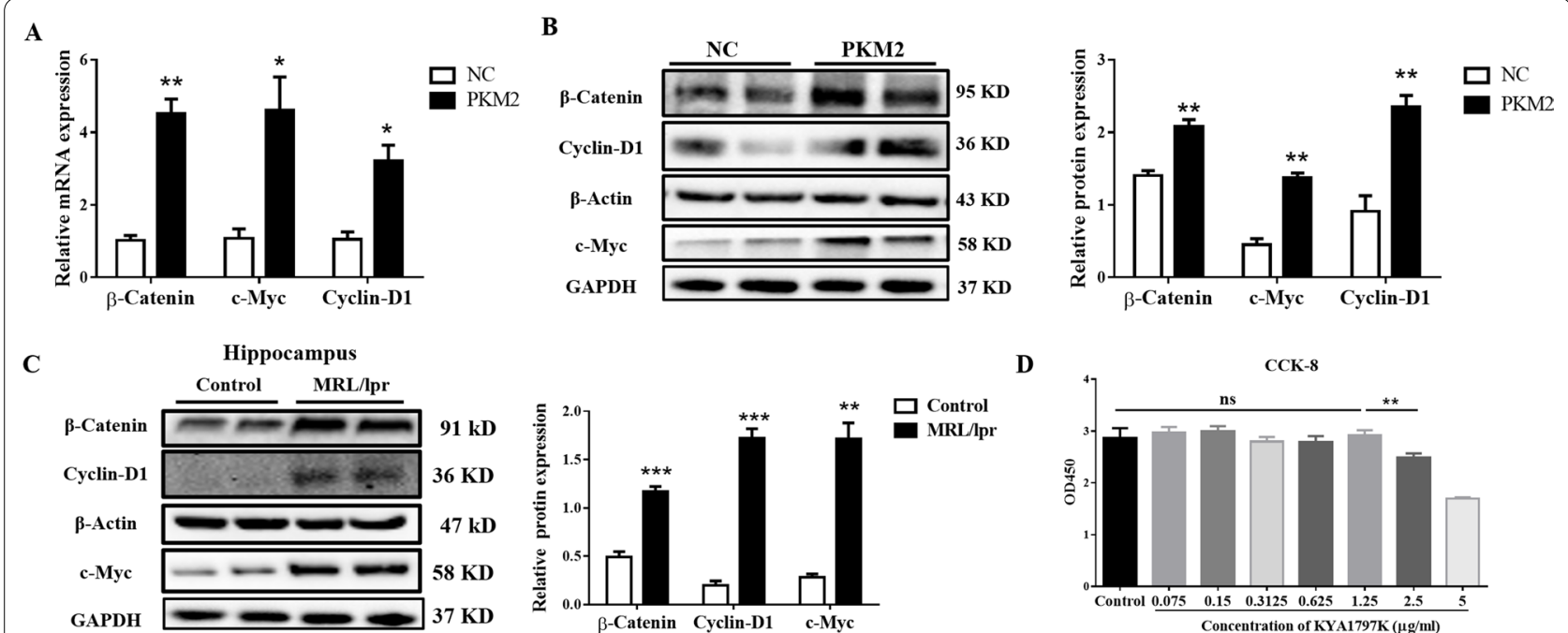

E

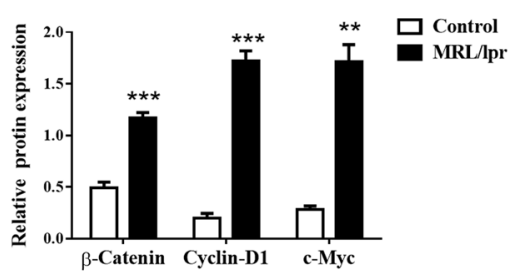

D

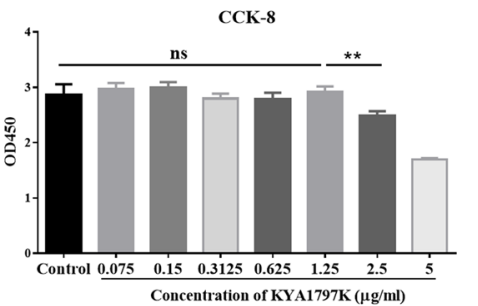

E

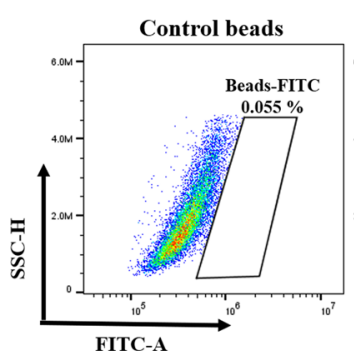

F

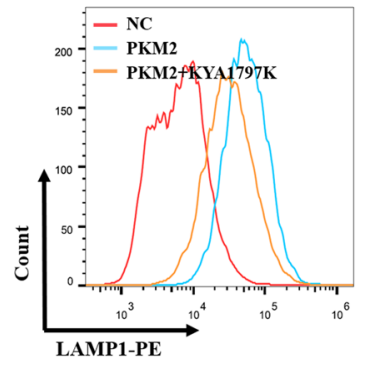

BV2 cells culture with latex beads-FITC

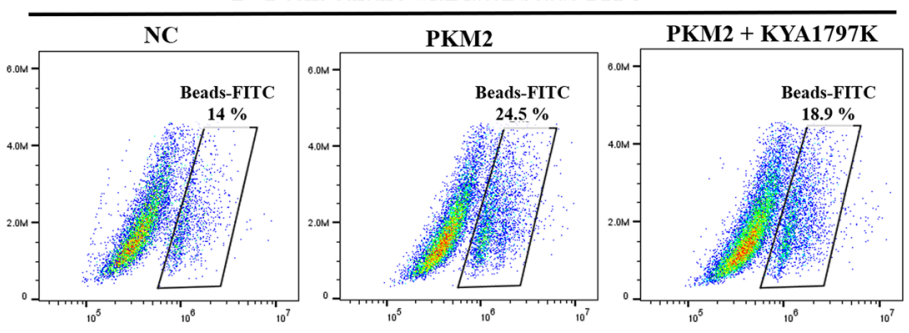

G
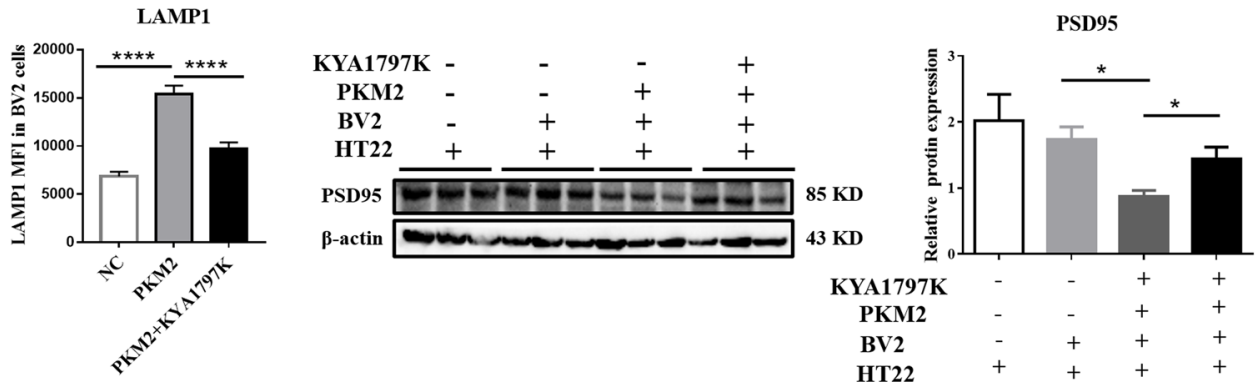

Fig. 7 PKM2 facilitates phagocytosis via the $\beta$-catenin signaling pathway. A The mRNA expression levels of $\beta$-catenin, $c-M y c$, and Cyclin-D1 detected by RT-PCR analysis at $24 \mathrm{~h}$ after plasmid transfection. B The protein expression of $\beta$-Catenin, c-Myc, and Cyclin-D1 detected by Western blot analysis $24 \mathrm{~h}$ after plasmid transfection. CWestern blot quantification of $\beta$-catenin, C-Myc, and Cyclin-D1 in the hippocampus of control and MLP/lpr mice, $n=4$. D BV2 cells were treated with different concentrations of $\beta$-catenin inhibitor (KYA1797K) for $24 \mathrm{~h}$. CCK8 detected the cytotoxicity of KYA1797K to BV2 cells. ETransient transfection of BV2 cells with the negative control plasmid (NC) or PKM2 over-expressed plasmid (PKM2). After transfection, BV2 cells were treated with or without $200 \mathrm{ng} / \mathrm{mL} \beta$-catenin inhibitor (KYA1797K) for $24 \mathrm{~h}$. Then, a phagocytic function test was performed, and the phagocytosis of BV2 cells was detected by flow cytometry. F Transient transfection of BV2 cells with NC or PKM2 over-expressed plasmid, $24 \mathrm{~h}$ later, BV2 cells were treated with or without $200 \mathrm{ng} / \mathrm{mL} \beta$-catenin inhibitor (KYA1797K) for $24 \mathrm{~h}$. The expression level of LAMP1 was detected by flow cytometry. G BV2 cells were transiently transfected with NC or PKM2 over-expressed plasmid (PKM2); 24 h later, BV2 cells were treated with or without $200 \mathrm{ng} / \mathrm{mL}$ KYA1797K for $24 \mathrm{~h}$, and then co-cultured with HT22 cells. Finally, a Western blot was used to analyze the protein level of PSD95. Data are presented as mean scores $\pm \mathrm{SEM},{ }^{*} P \leq 0.05,{ }^{* *} P \leq 0.01,{ }^{* * *} P \leq 0.001,{ }^{* * *} P \leq 0.0001 . n=3$ per group

overexpression was distinctly reversed after KYA1797K treated $(P \leq 0.05$, Fig. 7E). Furthermore, the expression of LAMP1 in BV2 cells was downregulated $(P \leq 0.05$, Fig. 7F), and the neuronal synapse phagocytosis of BV2 cells was weakened. Moreover, the application of
KYA1797K in BV2 cells stimulated by R848 also showed similar results (Additional file 1: Fig. S8E-G). Based on these findings, we concluded that inhibit $\beta$-catenin signaling pathway reversed the PKM2-induced excessive phagocytosis of microglial cells. 


\section{Microglial PKM2 inhibition alleviates brain damage and cognitive disorders in MRL/lpr mice}

Based on our above exploratory research, the AAV9shPKM2 virus, targeting microglia (Additional file 1: Fig. S9A), was stereotactically injected into the lateral ventricle of MRL/lpr mice to specifically downregulate PKM2 on microglia. After we verified the accuracy of targeting interfering PKM2 (Additional file 1: Fig. S9B), the H\&E staining showed that in microglial PKM2 inhibition group mice, the neurons in the hippocampal CA1, CA3, and DG brain regions were neatly arranged, the number of ghost cells was significantly reduced, and the injured neurons were significantly reduced (Fig. 8A). The neurophagy and microglial nodules were decreased in the cerebral cortex of the shPKM2 group compared to that of the empty vector group (Fig. 8A). Tunnel and NEUN costaining determined injured neurons. The results showed that the injured neurons in the shPKM2 group were significantly reduced (Fig. 8B).

Neuroinflammation and albumin deposition in the CNS are usually regarded as pathological manifestations of NPSLE [65]. IL-1 $\beta$ and IL-6 are usually used as

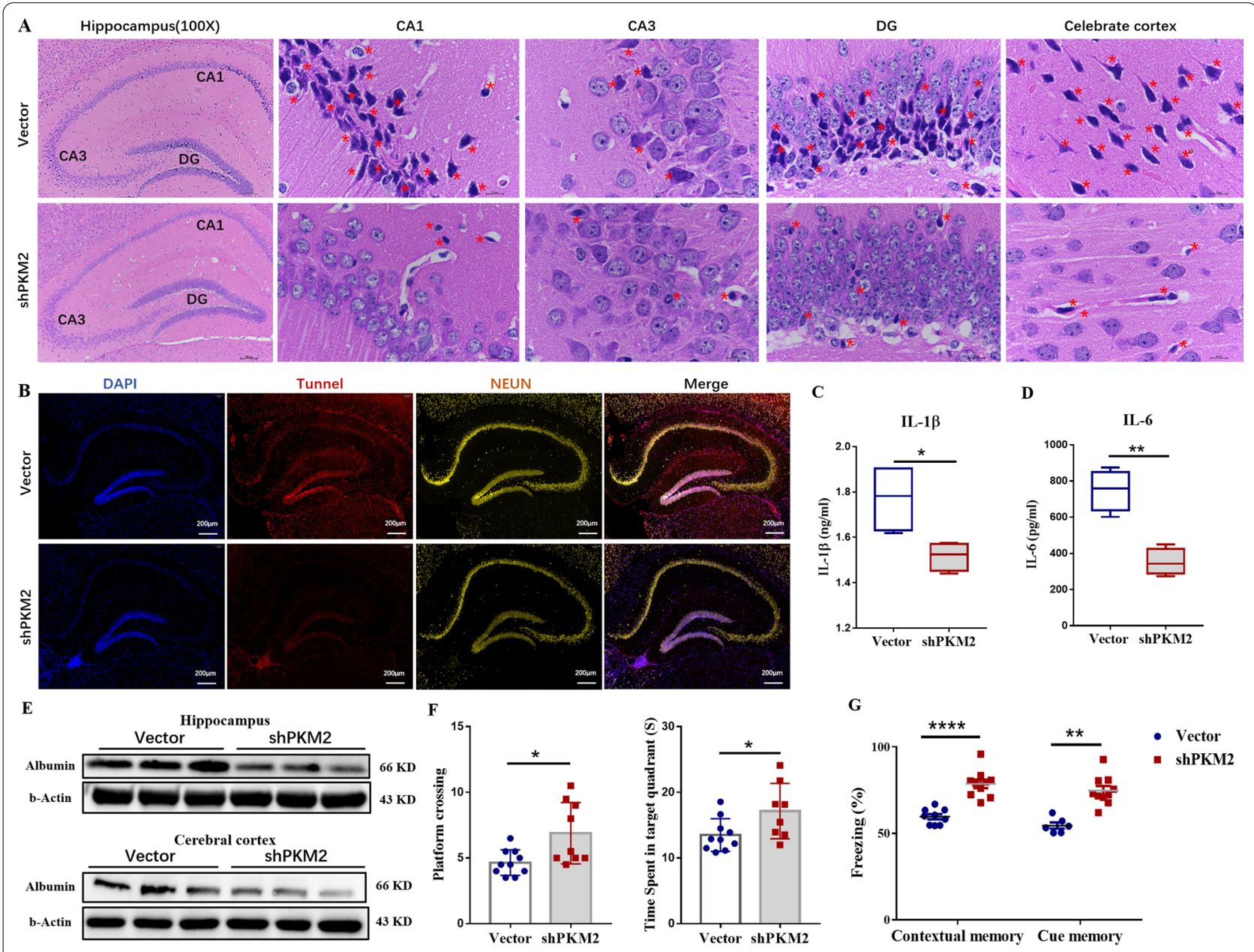

Fig. 8 Microglial PKM2 inhibition alleviates brain damage and cognitive disorders in MRL/lpr mice. Twenty female MRL/lpr mice, aged 8 weeks, were divided into two groups: vector group and shPKM2 group. The mice were maintained until week 10. Empty vector virus or AAV9-shPKM2 virus were then stereotactically injected into the lateral ventricle of MRL/lpr mice, until the mice reached week 20. A HE staining of the coronal area of the mouse brain of each group mice; Magnification: Hippocampus $(100 \times)$, CA1 $(1000 \times)$, CA3 $(1000 \times)$, DG $(1000 \times)$, celebrate cortex $(1000 \times)$, $n=4$. B Tunel (red) and NeuN (yellow) immunofluorescence double staining of the hippocampus of each group mice; bar $=200 \mu \mathrm{m}, n=4$. C The expression levels of IL-6 of the hippocampus of each group measured by ELISA, $n=6$. D The expression levels of IL-1 $\beta$ in the hippocampus of each group measured by ELISA, $n=6$. E Western blot quantification of albumin in the hippocampus and cortex, $n=4$. $\mathbf{F}$ On day 6 of the water maze experiment, the time they stayed in the target quadrant (left) and the number of times the mice crossed the platform (right) was recorded, $n=10$. G Fear conditioning tests. The percentage of the stiffness of mice was recorded during the test: different effects of 40- $\mathrm{Hz}$ light intervention, 2000- $\mathrm{Hz}$ sound intervention, and photoacoustic treatment on contextual and cue memory, respectively, $n=10$. Data are represented as mean $\pm S E M$, ${ }^{*} P \leq 0.05,{ }^{* *} P \leq 0.01,{ }^{* * *} P \leq 0.0001$ 
evaluation indicators of neuroinflammation in the hippocampus of MRL/lpr mice [66, 67]. Our results showed that the IL- 6 and IL-1 $\beta$ cytokine levels in the hippocampus tissue were downregulated after microglial PKM2 inhibition $(P \leq 0.05$, Fig. $8 C, D)$. Meanwhile, fewer albumin deposits in the hippocampus were detected (Fig. 8E). In summary, these data suggest that microglial PKM2 inhibition might relieve the brain injury of NPSLE mice.

Subsequently, MWM and conditional fear tests were applied to investigate the cognitive ability in treated NPSLE mice. Regardless of the time mice took to cross the platform or stay in the quadrant, mice in the shPKM2 group showed better results than the vector group (Fig. 8F and Additional file 1: Fig. S10A-C). Similarly, the percentage of rigidity in environmental fear and fear of sound stimulation in the shPKM2 group were higher than in the vector group (Fig. 8G). Thus, we speculated that microglial PKM2 inhibition in NPSLE mice contributes in ameliorating learning and memory dysfunction.

Furthermore, we observed that after silencing PKM2 on microglia, the number of hippocampal microglia was also decreased $(P \leq 0.01$, Fig. 9A), while the proinflammatory phenotype CD86 and LAMP1 expression on microglia were downregulated (Fig. 9B, C). At the same time,

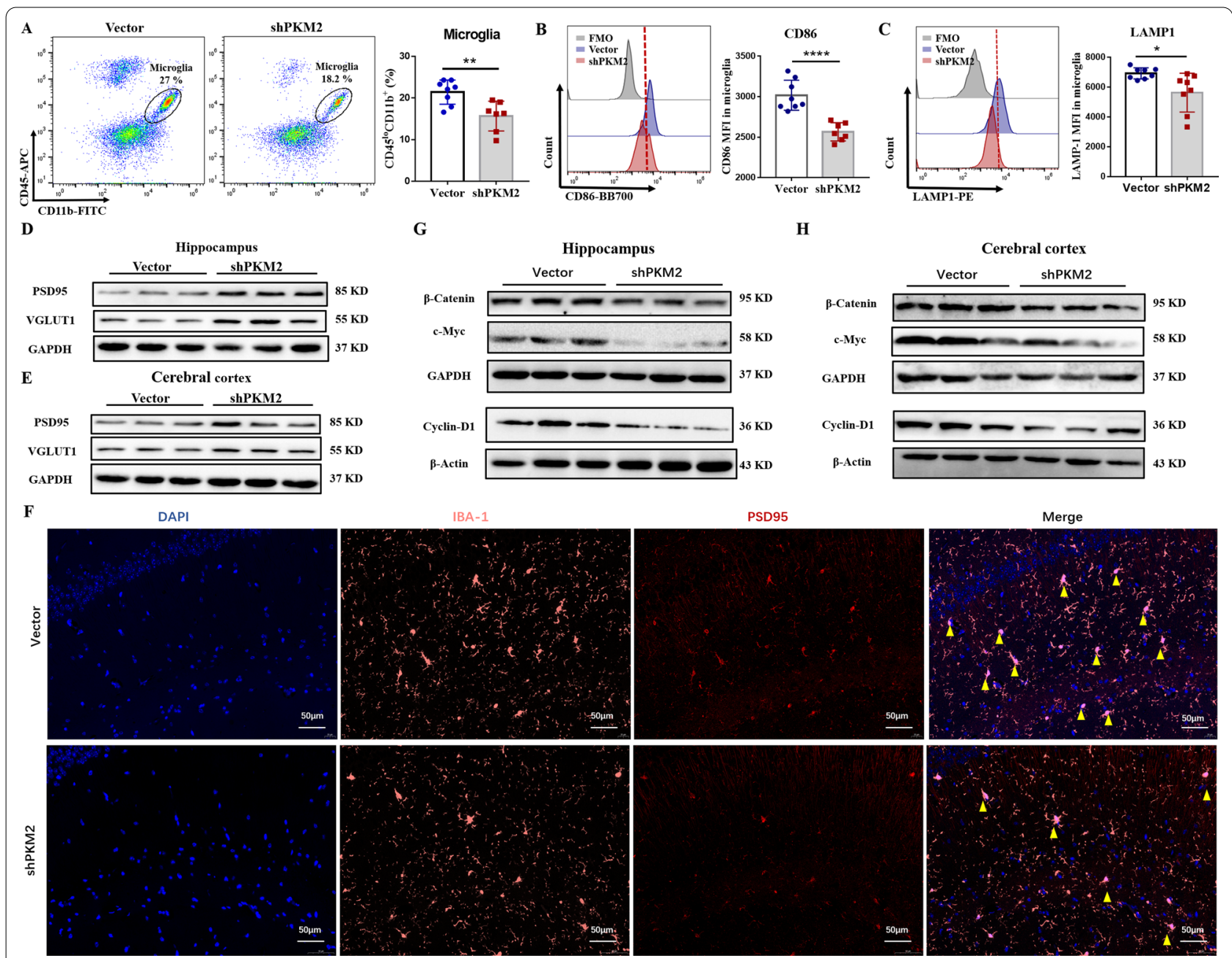

Fig. 9 Silencing microglial PKM2 reduces microglial activation and loss of neuronal synapses by $\beta$-catenin signaling pathway. A The percentage of microglia $\left(\mathrm{CD} 45^{\circ} \mathrm{CD} 11 \mathrm{~b}^{+}\right)$in the hippocampus, $n=6$. B The expression level of CD86 in microglia detected by flow cytometry, $n=6$. C The expression level of LAMP1 in microglia detected by flow cytometry, $n=6$. D Western blot quantification of PSD95 and VGLUT1 in the hippocampus of vector and sh-PKM2 group, $n=4$. E Western blot quantification of PSD95 and VGLUT1 in the cerebral cortex of vector and sh-PKM2 group, $n=4$. F PSD95 (red) and IBA-1 (pink) immunofluorescence double staining in the hippocampus of the mice in each group were detected by IF; $\operatorname{Bar}=200 \mu \mathrm{m}, n=4$. G Western blot quantification of $\beta$-catenin, c-Myc, and CyclinD1 in the hippocampus of vector and sh-PKM2, $n=4$. $\mathbf{H}$ Western blot quantification of $\beta$-catenin, $c-M y c$, and CyclinD1 in the cerebral cortex of vector and sh-PKM2, $n=4$. Data are represented as mean score \pm SEM, ${ }^{*} P \leq 0.05,{ }^{* *} P \leq 0.01,{ }^{* * *} P \leq 0.0001$ 
PSD95 and VGLUT1 expression levels in the shPKM2 group mice were obviously upregulated, compared to the empty vector group (Fig. 9D, E). Immunofluorescence staining also showed less colocalization on IBA-1 (microglia marker) and PSD95 (neuronal synaptic marker) in the shPKM2 group (Fig. 9F). These data indicated that knocking-down PKM2 on microglia markedly suppresses the synaptic pruning of neurons in lupus mice. In addition, after microglial PKM2 inhibition, the protein levels of $\beta$-catenin, c-Myc, and Cyclin-D1 were significantly downregulated (Fig. 9G, H). In brief, this data suggested that microglial PKM2 inhibition alleviates cognitive disorders and brain damage in MRL/lpr mice and reduces microglial activation and loss of neuronal synapses by blocking the $\beta$-catenin signaling pathway.

\section{Discussion}

This study showed that PKM2 was upregulated in lupus microglia with over-activated glycolysis in the hippocampus. PKM2 positively regulates the phagocytosis of microglia by the $\beta$-catenin signaling pathway, which aggravates the loss of neuronal synapses, and promotes the occurrence of cognitive dysfunction in NPSLE mice. Inhibition of the expression of microglial PKM2 could relieve the above symptoms. These results indicated that PKM2 might be a new target for the treatment of cognitive dysfunction in NPSLE.

Several factors, such as cerebrospinal fluid (CSF), IL-6 levels, and interferon-alpha (IFN- $\alpha$ ), were recently reported to be closely related to the development of NPSLE [68]. In addition, autoantibodies and RNA-protein antigens form immune complexes in CSF and initiate the proinflammatory cascade [69]. However, the exact cause is not entirely clear. In patients with neuropsychiatric SLE, hippocampal structural lesions have been identified [51]. Similarly, structural abnormalities in the hippocampus of MRL/lpr mice have also been reported by Sakic et al. [52]. Unsurprisingly, our results revealed that IL- 6 and IL- $1 \beta$ cytokine levels in the hippocampus of lupus mice were significantly higher than those in the control mice, with albumin accumulation in situ (data not shown). Besides, B cells chronically stimulated with B-cell activating factor, a cytokine associated with SLE, show enhanced glycolysis and subsequently synthesize more antibodies [70, 71]. Increased glycolysis has also been linked to mitochondrial oxidative stress in lupusprone mice [72]. T cells exhibit persistent mitochondrial hyperpolarization due to increased mitochondrial reactive oxygen species production, depletion of reduced glutathione, and diminished mitochondrial ATP synthesis, which predisposes to proinflammatory death by necrosis [73]. These phenomena indicate that the metabolism reconfiguration might influence the pathogenesis of SLE
$[74,75]$. Interestingly, several neuroimaging reports indicated an association between cerebral hypometabolism and NPSLE [10, 11, 76], prompting us to further explore hippocampal metabolic abnormalities in MRL/lpr lupus mice.

In this study, we extracted the metabolites in the hippocampal tissues and conducted metabolomics analysis (LC-MS analysis), which exhibited abnormal glycolysis metabolites in the hippocampus of MRL/lpr mice. Under normal oxygen conditions, cells obtain energy via mitochondria oxidative phosphorylation and glycolysis. The activities and metabolic flux of the two pathways are delicately tuned to providing metabolic precursors for biosynthesis and energy production [77, 78]. Glucose consumption and lactic acid production in the hippocampus and cortex of MRL/lpr mice increase. Moreover, studies have indicated that active brain tissue does not fully oxidize glucose, but instead generates a local surplus of lactate, a phenomenon termed aerobic glycolysis $[79,80]$. It remains to be clarified why is it important to engage in inefficient ATP production by glycolysis when energy demand is highest, and oxygen is plentiful. Substantial in vitro and in vivo evidence have indicated that neurons, the main energy consumers of brain tissue, could consume both glucose and lactate; the latter is produced by glycolysis [81-84]. Therefore, we speculated that even in the case of sufficient oxygen, there is an abnormal increase of aerobic glycolysis in the lupus brain to provide sufficient energy donors. Apart from this, in classically activated M1 macrophages, metabolism is shifted towards glycolysis. This switch increases glucose uptake and lactate production with activation of the pentose phosphate pathway and decreased mitochondrial oxygen consumption [80, 85, 86]. Microglia, the resident macrophages in the brain, revealed M1 polarization in the hippocampus of MRL/lpr mice, which might be one of the factors causing increased glucose consumption and lactate production in our results.

Glycolysis is the metabolic pathway that converts glucose into pyruvate, controlled by various glycolytic enzymes [87]. PKM2 is an important rate-limiting enzyme in glycolysis that catalyzes the irreversible conversion of phosphoenolpyruvate to pyruvate. This phenomenon could fuel the tricarboxylic acid cycle or convert pyruvate to lactate, which is then secreted [88]. PKM2 is highly expressed in cancer cells, activated immune cells [89, 90], and upregulated in most human cancers [91]. Over recent years, the regulatory role of PKM2 in autoimmune diseases has gained increasing attention. Previous studies have shown that pharmacological activation of PKM2 inhibits $\mathrm{CD} 4^{+} \mathrm{T}$ cell pathogenicity and suppresses experimental autoimmune encephalomyelitis [92]. PKM2-mediated aerobic 
glycolysis contributes to macrophage activation and inflammatory response, while PKM2 inhibitor protects the mice from lethal endotoxemia and sepsis [93]. Our current data indicated that PKM2 was abnormally expressed on the hippocampal microglia of MRL/lpr mice. However, in hypoxic-ischemic encephalopathy in neonatal rats, double IF labeling showed that PKM2 was mainly localized in the neurons of the ipsilateral cerebral cortex and not in astrocytes or microglia [94]. Some studies revealed that early recombinant PKM2 (rPKM2) treatment exerts an acute neuroprotective effect against ischemic brain damage, whereas delayed rPKM2 treatment promotes regenerative activities in the poststroke brain, thereby promoting functional recovery [95]. These phenomena suggested that PKM2 could be a target for the treatment of inflammatory brain diseases. Nevertheless, few studies have reported on the conformational differences of PKM2 in these inflammatory brain diseases.

It has been reported that PKM2 has both a dimeric form and a tetrameric form [53-55]. The biological effects between the tetramer and the dimer are significantly different $[56,57]$. When PKM2 is in a tetrameric state, it has a higher affinity with its substrate phosphoenolpyruvate (PEP) and a higher PK enzymatic activity to catalyze the production of pyruvate by PEP [96-98]. After the tetramer is converted into a dimer, PKM2 can exist in a variety of different intracellular localization, and enter the nuclear to regulate gene expression [99]. PKM2 can also be modified with phosphorylation, acetylation, and other proteins to regulate protein activity and intracellular localization [100]. Our result showed that tetrameric and dimeric formation of PKM2 was enhanced in the hippocampus of MRL/lpr mice compared to control mice. Moreover, after R848 treatment, total PKM2 in BV2 cells were over-expressed while cytoplasmic PKM2 decreased, thus suggesting that PKM2 enters the nucleus under R848 stimulation. Similarly, the immunofluorescence more intuitively confirmed this phenomenon. All these results suggested that both dimer and tetramer of PKM2 participated in the development of NPSLE.

Microglia serve as first responders to neuronal damage and infections to restore/maintain homeostasis and have a critical role in neural circuit connectivity [101]. Previous studies have shown histological evidence of perivascular microglial activation $[102,103]$ in SLE patients and SLE mouse models based on positive microglial staining of CD68 and Iba-1 antigens [104]. Damaged neurons caused by synapse loss are closely associated with anomalous cognition, mainly learning and memory dysfunction, which is regulated by the hippocampus $[105,106]$. In a recent study, excessive synaptic pruning by microglia was associated with the behavioral deficits of the 564Igi SLE mouse model, which displayed anxiety and cognitive defects [107]. Supposedly, microglia depletion ameliorated disease in multiple models of NPSLE [108]. Herein, we found that microglia proliferated and were activated in the hippocampus and cortex of MRL/lpr mice. Meanwhile, microglial phagocytosis was enhanced, thereby resulting in the loss of neurons and the occurrence of MRL/lpr cognitive dysfunction. We speculated that this might be related to the abnormal expression of PKM2 on these microglia. To better prove the correlation between microglial PKM2 upregulation and hippocampal neuron damage or cognitive dysfunction in lupus, AAV9shPKM2 virus, targeting microglia, was stereotactically injected into the lateral ventricle of MRL/lpr mice to specifically downregulate PKM2 on microglia, after which morphological alterations of neurons in the hippocampus were examined, and hippocampus-dependent spatial learning and memory and contextual memory were also analyzed by MWM and fear-conditioning paradigm [109, 110]. The results showed that microglial PKM2 inhibition alleviated cognitive disorders and brain damage in MRL/ lpr mice.

In addition to brain damage and cognitive improvement after microglial PKM2 inhibition, the expression of IL- 6 and IL- $1 \beta$ in the hippocampus of MRL/lpr mice, were also reversed. It has been reported that increased serum and hippocampal levels of IL- 6 and IL- $1 \beta$ are consistent with the progression of the lupus-like disease $[111,112]$. IL- 6 and IL- $1 \beta$ alter emotional reactivity and clinical behavior, provoking sickness and induce impairments in spatial learning experimentally $[66,67]$; therefore, they could be used as evaluation indicators of neuroinflammation in the hippocampus of MRL/lpr mice. The downregulation of IL- 6 and IL- $1 \beta$ in the hippocampus following AAV9-shPKM2 administration in MRL/lpr mice indicated that microglial PKM2 inhibition could improve neuroinflammation in MRL/lpr mice.

CNS lupus is a heterogeneous disease with many symptoms and causes. Our data suggested that the upregulation of PKM2-mediated glycolysis enhances the activation and phagocytosis of microglia, resulting in the loss of neuronal synapses and inducing cognitive dysfunction in lupus. Together, these findings suggested a novel mechanism for CNS lupus and provided a rationale for expanding future clinical trials to include CNS lupus patients, especially those with detectable glycolysis signatures.

\section{Conclusions}

Our data suggest that PKM2 has an important role in the development of cognitive impairment in NPSLE mice. PKM2 upregulated glycolysis in the brain tissue of NPSLE mice and increased the activation of microglia and the ability of phagocytizing neuronal synapses, leading to 
neuronal loss and cognitive dysfunction in lupus mice. Inhibiting the expression of microglial PKM2 alleviated cognitive impairment and brain damage in NPSLE mice. These phenomena indicated that PKM2 might become a potential therapeutic target for the treatment of lupus encephalopathy.

\begin{abstract}
Abbreviations
NPSLE: Neuropsychiatric systemic lupus erythematosus; PKM2: Pyruvate kinase isoform M2; SLE: Systemic lupus erythematosus; DNRAb: Anti-DNA antibodies; CNS: Central nervous system; BSA: Bovine serum albumin; DAPI: 2-(4-Amidinophenyl)-1H-indole-6-carboxamidine; SDS-PAGE: Sodium dodecyl sulfate-polyacrylamide gel electrophoresis; IMQ: Imiquimod; ELISA: Enzymelinked immunosorbent assay; MWM: Morris water maze; PGI: Phosphoglucose isomerase; PFK: Phosphofructokinase-1; DHAP: Dihydroxyacetone phosphate; TPI: Triosephosphate isomerase; HK1: Hexokinase 1; PFKFB3: 6-Phosphofructo2-kinase/fructose-2,6-bisphosphatase 3; ENO1: Enolase 1; LDHA: Lactate dehydrogenase A; GLUT1: Glucose transporter 1; LDHA: Lactic dehydrogenase kinase A; PDK1: Pyruvate dehydrogenase kinase 1; LAMP1: Lysosomeassociated membrane protein 1; VGLUT1: Vesicular glutamate transporter 1; CA: Cornu ammonis; DG: Dentate gyrus; BBB: Brain blood-brain barrier; CSF: Cerebrospinal fluid; MFI: Mean fluorescence intensity.
\end{abstract}

\section{Supplementary Information}

The online version contains supplementary material available at https://doi. org/10.1186/s12974-021-02279-9.

Additional file 1: Supplemental figures. Fig. S1. PCA model score scatter plot and OPLS-DA model show the separation of glycolysis metabolites in hippocampus of control mice (blue) and MLR/lpr group mice (red). All samples were included in this analysis. The horizontal ordinate (PC [1]) indicates the first principal component, and the vertical ordinate (PC [2]) indicates the second principal component. Fig. S2. The glycolytic activity of hippocampus and cortex of IMQ-induced mice might be related to the high expression of PKM2. (A) Glucose levels and lactate production in the hippocampus and cerebral cortex of IMQ-induced mice were normalized to that of the control group. (B) The mRNA expression of GLUT1, LDHA, and PDK1 in the hippocampus of control and IMQ-induced groups. (C) The mRNA expression of GLUT1, LDHA, and PDK1 in the celebrate cortex of IMQ-induced mice to the control group mice. Data represent the mean scores \pm SEM. ${ }^{*} P \leq 0.05,{ }^{* *} P \leq 0.01,{ }^{* * *} P \leq 0.001 . n=6$ mice per group. Fig. S3. The expression of PKM2 increases in IMQ-induced mice. (A) The mRNA expression of PKM2, HK1, PFKFB3, and ENO1 in hippocampus of IMQ-induced mice to the control group mice. (B) The mRNA expression of PKM2, HK1, PFKFB3, and ENO1 in celebrate cortex of in IMQ-induced mice to the control group mice. Data represent the mean scores \pm SEM. ${ }^{*} P \leq 0.01 . n=6$ mice per group. Fig. S4. The expression of dimeric and tetrameric forms of PKM2. (A) The abundance of the monomeric, dimeric and tetrameric forms of PKM2 are presented by the image with a short exposure (30 s). (B) The monomeric and dimeric forms are best visualized by the image with a long exposure (100 s). (C) Western blot quantification of total PKM2 and cytoplasmic PKM2 in BV2 cells treated or not treated with R848 agonist. (D) The expression levels of PKM2 (green), DAPI (blue), and their colocalization in BV2 cells treated or not treated with R848 agonist by IF staining; Bar $=20 \mu \mathrm{m}$. Fig. S5. Experimental procedure of flow cytometry analysis. (A) The schematic of sample preparation before flow cytometry detection of mouse hippocampal microglia. (B) Gating strategy of microglia $\left(\mathrm{CD}^{5} 5^{\mathrm{lo}} \mathrm{CD} 11 \mathrm{~b}^{+}\right)$in the hippocampus by flow cytometry. Fig. S6. Microglia is activated and PKM2 is highly expressed in hippocampal tissue of IMQ-induced mice. (A) The percentage of microglia $\left(\mathrm{CD} 45^{\mathrm{lo}} \mathrm{CD} 11 \mathrm{~b}^{+}\right)$in the hippocampus of IMQ-induced mice to the control group mice. (B) The expression level of CD86 in microglia of IMQ-induced mice to the control group mice were detected by flow cytometry. (C) The expression level of LAMP1 in the microglia of control and IMQ-induced mice t by flow cytometry. (D) The expression levels of CD68 (green), IBA-1 (red), and their colocalization (yellow) in the hippocampus of control and IMQ-induced mice were detected by IF staining, Bar $=20 \mu \mathrm{m}$. (E) WB quantification of IBA-1 and CD68 in hippocampus of IMQ-induced mice and control group mice. (F) The mRNA expressions of PKM2 in the hippocampal of IMQ-induced mice and control group mice. (G) WB quantification of PKM2 in hippocampus of IMQ-induced mice and control group mice. Data represent the mean scores \pm SEM. ${ }^{*} P \leq 0.05,{ }^{* *} P \leq 0.01$. $n=6$ mice per group. Fig. S7. TLR7 activator enhances phagocytic activity of microglia and the nuclear transfer of PKM2. (A) The expression level of LAMP1 was detected by flow cytometry with or without R848 treated for $24 \mathrm{~h}$. (B) Western blot quantification of LAMP1 in BV2 cells treated with different concentrations of R848 (50, 100, $200 \mathrm{ng} / \mathrm{ml})$ for $24 \mathrm{~h}$. (C) Phagocytic function test. BV2 cells treated with different concentrations of R848 $(50,100,200 \mathrm{ng} / \mathrm{ml})$ for $24 \mathrm{~h}$, then BV2 cells cultured with DMEM which contained fluorescent beads in incubator for $2 \mathrm{~h}$. Collected the BV2 cells and detected by flow cytometry. (D) BV2 cells treated with different concentrations of R848 $(50,100 \mathrm{ng} / \mathrm{ml})$ for $24 \mathrm{~h}$. Western blot analysis of the protein expression of PSD95 after HT22 cells co-cultured with different treated BV2 cells. (E) BV2 cells treated with different concentrations of R848 $(50,100,200 \mathrm{ng} / \mathrm{ml})$ for $24 \mathrm{~h}$, then the mRNA expressions of PKM2 were detected by RT-qPCR. (F) BV2 cells treated with different concentrations of R848 $(50,100,200 \mathrm{ng} / \mathrm{ml})$ for $24 \mathrm{~h}$, then the protein expressions of PKM 2 were detected by western blotting. (G) BV2 cells treated with R848 (100 ng/ml) for different times $(12 \mathrm{~h}, 24 \mathrm{~h}, 36 \mathrm{~h})$, then the mRNA expressions of PKM2 were detected by RT-qPCR. Data represent the mean scores \pm SEM. ${ }^{*} P \leq 0.05,{ }^{* *} P \leq 0.01,{ }^{* * *} P \leq 0.001 . n=3$. Fig. S8. TLR7 activates the PKM2/b-catenin pathway to enhance the phagocytic activity of microglia. (A) The mRNA expression level of $c-M y c, A x i n-2$ and Cyclin-D1 were detected by RT-qPCR in BV2 cells treated with $100 \mathrm{ng} / \mathrm{ml}$ R848 for different time (12 h, $24 \mathrm{~h}, 72 \mathrm{~h}$ ), and analyzed the correlation between PKM2 and c-Myc, Axin-2 and Cyclin-D1. (B) The mRNA expression level of C-Myc, Axin-2 and Cyclin-D1 were detected by RT-qPCR in BV2 cells treated with different concentrations of R848 (50, 100, $200 \mathrm{ng} / \mathrm{ml})$ for $24 \mathrm{~h}$, and analyzed the correlation between PKM2 and C-Myc, Axin-2 and Cyclin-D1. (C) Western blot quantification of $\beta$-Catenin, c-Myc and Cyclin-D1 in BV2 cells treated with different concentrations of $\operatorname{R848}(50,100,200 \mathrm{ng} / \mathrm{ml})$ for $24 \mathrm{~h}$. (D) The expression levels of $\beta$-Catenin (Green) in BV2 cells treated with $100 \mathrm{ng} / \mathrm{ml}$ R848 by if staining, Bar $=20 \mu \mathrm{m}$. (E) Phagocytic function test. BV2 cells induced with $100 \mathrm{ng} / \mathrm{ml}$ R848 for $24 \mathrm{~h}$, then treated with or without $200 \mathrm{ng} / \mathrm{ml}$ B-Catenin inhibitor (KYA1797K) for $24 \mathrm{~h}$. All group BV2 cells were cultured with DMEM which contained fluorescent beads in incubator for $2 \mathrm{~h}$. Collected the BV2 cells and detected by flow cytometry. (F) BV2 cells induced with $100 \mathrm{ng} / \mathrm{ml}$ R848 for $24 \mathrm{~h}$, then treated with or without $200 \mathrm{ng} / \mathrm{ml}$ B-Catenin inhibitor (KYA1797K) for $24 \mathrm{~h}$. The expression level of LAMP1 in BV2 cells was detected by flow cytometry. (G) BV2 cells induced with $100 \mathrm{ng} / \mathrm{ml} \mathrm{R} 848$ for $24 \mathrm{~h}$, then treated with or without $200 \mathrm{ng} / \mathrm{ml} \beta$-Catenin inhibitor (KYA1797K) for $24 \mathrm{~h}$. Western blot analysis of the protein expression of PSD95 after HT22 cells co-cultured with different treated BV2 cells. Data represent the mean scores \pm SEM. ${ }^{*} P \leq 0.05$, ${ }^{* *} P \leq 0.01,{ }^{* * *} P \leq 0.001 . n=3$. Figure S9. Verification of PKM2 conditional knockout. (A) Schematic diagram of virus structure. (B) The expression levels of PKM2 (red), GFAP (white), and their colocalization in the hippocampus detected by IF staining, $\mathrm{Bar}=20 \mu \mathrm{m}$. (C) The expression levels of PKM2 (red), NeuN (white), and their colocalization in the hippocampus detected by IF staining, Bar $=20 \mu \mathrm{m}$. (D) The expression levels of PKM2 (red), IBA-1 (white), and their colocalization in the hippocampus of vector group and shPKM2 group mice detected by IF staining, Bar $=20 \mu \mathrm{m}$. Fig. S10. Route of mouse water maze. (A) Morris water maze test. Representative swimming traces of mice from different groups on the first day and the fifth training day. The hidden platform is located in quadrant III. (B) The time latency to find the hidden platform in different groups of mice during consecutive 5 training days. (C) The swimming trajectory of the test mice on day 6 of the water maze experiment.

\section{Acknowledgements}

We would like to thank Prof. Tianjiao Xia (Medical school, Nanjing University) and Dr. Lizhi Xu (Medical school, Nanjing University) for helpful guidance on behavioral testing. We also thank Shanghai Biotree Biotechnology Ltd., for the assistance in metabolomics data acquisition and data analysis. 


\section{Authors' contributions}

$H D, Y H, J L$, and $Y S$ conceived and designed the project. $L L, H W, X L$, and $X Q$ performed the experiments. $L L, H W$, LT, and JN analyzed the data. LL wrote the manuscript, and HD revised the manuscript. All authors read and approved the final manuscript.

\section{Funding}

The present work was supported by the National Natural Science Foundation of China (32070883, 31872732, and 81871319), Jiangsu Province Six Talent Peaks Project (YY-021), Jiangsu Province key Social and Development R\&A Grant (BE2019706 and BE2019617), Jiangsu Provincial Medical Youth Talent (QNRC2016005), and Research Project of Jiangsu Province Health Committee ( H2019060)

\section{Availability of data and materials}

The datasets used and/or analyzed during the current study are available from the corresponding author on reasonable request.

\section{Declarations}

\section{Ethics approval and consent to participate}

All animal experiments complied with the protocols approved by the Jiangsu Provincial Animal Care. The experiments on mice were approved by Institutional Animal Care and Use Committee, Nanjing University, and all experiments were performed in accordance with relevant guidelines and regulations.

\section{Consent for publication}

Not applicable.

\section{Competing interests}

The authors declare that they have no competing interests.

\section{Author details}

'The State Key Laboratory of Pharmaceutical Biotechnology, Division of Immunology, Medical School, Nanjing University, Nanjing 210093, People's Republic of China. ${ }^{2}$ Jiangsu Key Laboratory of Molecular Medicine, Nanjing 210093, People's Republic of China. ${ }^{3}$ Department of Rheumatology and Immunology, Nanjing Drum Tower Hospital, The Affiliated Hospital of Nanjing University Medical School, Nanjing 210008, People's Republic of China. ${ }^{4}$ The State Key Laboratory of Pharmaceutical Biotechnology and Collaborative Innovation Center of Chemistry for Life Sciences, School of Life Sciences, Nanjing University, Nanjing 210023, China

Received: 4 December 2020 Accepted: 31 August 2021

Published online: 13 October 2021

\section{References}

1. Hanly JG. Diagnosis and management of neuropsychiatric SLE. Nat Rev Rheumatol. 2014;10(6):338-47.

2. Carter EE, et al. The global burden of SLE: prevalence, health disparities and socioeconomic impact. Nat Rev Rheumatol. 2016;12(10):605-20.

3. Schwartz N, Stock AD, Putterman C. Neuropsychiatric lupus: new mechanistic insights and future treatment directions. Nat Rev Rheumatol. 2019;15(3):137-52

4. The American College of Rheumatology nomenclature and case definitions for neuropsychiatric lupus syndromes. Arthritis Rheum. 1999;42(4):599-608

5. Li X, Xiang X, Sun J, et al. Prevalence, outcome and prognostic factors of neuropsychiatric systemic lupus erythematosus: a real world single center study. Mod Rheumatol. 2020;30(2):321-6.

6. Olah C, Schwartz N, Denton C, et al. Cognitive dysfunction in autoimmune rheumatic diseases. Arthritis Res Therapy. 2020. https://doi.org/ 10.1186/s13075-020-02180-5.

7. Yue R, Gurung I, Long XX, et al. Prevalence, involved domains, and predictor of cognitive dysfunction in systemic lupus erythematosus. Lupus. 2020. https://doi.org/10.1177/0961203320958061.
8. Mink JW, Blumenschine RJ, Adams DB. Ratio of central nervous system to body metabolism in vertebrates: its constancy and functional basis. Am J Physiol. 1981;241(3):R203-212.

9. Ivanisevic J, Siuzdak G. The role of metabolomics in brain metabolism research. J Neuroimmune Pharmacol. 2015;10(3):391-5.

10. Weiner SM, Otte A, Schumacher $M$, et al. Diagnosis and monitoring of central nervous system involvement in systemic lupus erythematosus: value of F-18 fluorodeoxyglucose PET. Ann Rheum Dis. 2000:59:377-85.

11. Lee SW, Park MC, Lee SK, et al. The efficacy of brain (18)F-fluorodeoxyglucose positron emission tomography in neuropsychiatric lupus patients with normal brain magnetic resonance imaging findings. Lupus. 2012;21:1531-7.

12. Alexander JJ, Zwingmann C, Quigg R. MRL/lpr mice have alterations in brain metabolism as shown with [1H-13C] NMR spectroscopy. Neurochem Int. 2005:47(1-2):143-51.

13. Zhang Z, Wang $Y$, Shen Z, et al. The neurochemical and microstructural changes in the brain of systemic lupus erythematosus patients: a multimodal MRI study. Sci Rep. 2016;6:19026.

14. Camandola S, Mattson MP. Brain metabolism in health, aging, and neurodegeneration. EMBO J. 2017;36(11):1474-92.

15. Vo A, Volpe BT, Tang CC, et al. Regional brain metabolism in a murine systemic lupus erythematosus model. J Cereb Blood Flow Metab. 2014;34(8):1315-20.

16. Zhao C-H, Li G-H, Wang Q, et al. Mechanisms of propofol attenuation of ketamine-induced neonatal brain injury. Eur Rev Med Pharmacol Sci. 2016;20(1):133-7.

17. Butterfield DA, Poon HF, St Clair D, et al. Redox proteomics identification of oxidatively modified hippocampal proteins in mild cognitive impairment: insights into the development of Alzheimer's disease. Neurobiol Dis. 2006:22(2):223-32.

18. Venkatesan A, Uzasci L, Chen Z, et al. Impairment of adult hippocampal neural progenitor proliferation by methamphetamine: role for nitrotyrosination. Mol Brain. 2011;4:28.

19. Gomez Perdiguero E, Schulz C, Geissmann F. Development and homeostasis of "resident" myeloid cells: the case of the microglia. Glia. 2013;61(1):112-20.

20. Prinz M, Priller J. Microglia and brain macrophages in the molecular age: from origin to neuropsychiatric disease. Nat Rev Neurosci. 2014;15(5):300-12.

21. Reeves WH, Lee PY, Weinstein JS, et al. Induction of autoimmunity by pristane and other naturally occurring hydrocarbons. Trends Immunol. 2009;30(9):455-64.

22. Wolf SA, Boddeke HW, Kettenmann H. Microglia in physiology and disease. Annu Rev Physiol. 2017;79:619-43.

23. Hong S, Dissing-Olesen L, Stevens B. New insights on the role of microglia in synaptic pruning in health and disease. Curr Opin Neurobiol. 2016;36:128-34

24. Parkhurst CN, Yang G, Ninan I, et al. Microglia promote learningdependent synapse formation through brain-derived neurotrophic factor. Cell. 2013;155(7):1596-609.

25. Udeochu JC, Shea JM, Villeda SA. Microglia communication: parallels between aging and Alzheimer's disease. Clin Exp Neuroimmunol. 2016;7(2):114-25.

26. Streit WJ, Xue QS, Tischer J, et al. Microglial pathology. Acta Neuropathol Commun. 2014;2:142.

27. Sarlus H, Heneka MT. Microglia in Alzheimer's disease. J Clin Invest. 2017;127(9):3240-9.

28. Lecours C, Bordeleau M, Cantin L, et al. Microglial implication in Parkinson's disease: loss of beneficial physiological roles or gain of inflammatory functions? Front Cell Neurosci. 2018;12:282.

29. Geloso MC, Corvino V, Marchese $\mathrm{E}$, et al. The dual role of microglia in ALS: mechanisms and therapeutic approaches. Front Aging Neurosci. 2017:9:242

30. Nomura A, Noto D, Murayama G, et al. Unique primed status of microglia under the systemic autoimmune condition of lupus-prone mice. Arthritis Res Therapy. 2019;21(1):303.

31. Haruwaka K, Ikegami A, Tachibana Y, et al. Dual microglia effects on blood brain barrier permeability induced by systemic inflammation. Nat Commun. 2019;10(1):5816. 
32. Kong X, Zhang Z, Fu T, et al. TNF-alpha regulates microglial activation via the NF-kappaB signaling pathway in systemic lupus erythematosus with depression. Int J Biol Macromol. 2019;125:892-900.

33. Wen J, Chen $\mathrm{CH}$, Stock $A$, et al. Intracerebroventricular administration of TNF-like weak inducer of apoptosis induces depression-like behavior and cognitive dysfunction in non-autoimmune mice. Brain Behav Immun. 2016;54:27-37.

34. Nestor J, Arinuma Y, Huerta TS, et al. Lupus antibodies induce behavioral changes mediated by microglia and blocked by ACE inhibitors. J Exp Med. 2018;215(10):2554-66.

35. Piras BA, Tian $Y, X u Y$, et al. Systemic injection of AAV9 carrying a periostin promoter targets gene expression to a myofibroblast-like lineage in mouse hearts after reperfused myocardial infarction. Gene Ther. 2016;23(5):469-78.

36. Yang Y, Wang C, Cheng P, et al. CD180 ligation inhibits TLR7- and TLR9mediated activation of macrophages and dendritic cells through the Lyn-SHP-1/2 Axis in murine lupus. Front Immunol. 2018;9:2643.

37. Eykens C, Rossaert E, Duqué S, et al. AAV9-mediated gene delivery of MCT1 to oligodendrocytes does not provide a therapeutic benefit in a mouse model of ALS. Mol Therapy Methods Clin Dev. 2021;20(20):508-19.

38. Shi R, Zhang J, Fang B, et al. Runners' metabolomic changes following marathon. Nutr Metab (Lond). 2020;13(17):19.

39. Hao R, Xiaodong Du, Yang C, et al. Integrated application of transcriptomics and metabolomics provides insights into unsynchronized growth in pearl oyster Pinctada fucata martensii. Sci Total Environ. 2019;20(666):46-56

40. Frank MG, et al. Rapid isolation of highly enriched and quiescent microglia from adult rat hippocampus: immunophenotypic and functional characteristics. J Neurosci Methods. 2006;151(2):121-30.

41. Wan H, Wang Q, Chen X, et al. WDR45 contributes to neurodegeneration through regulation of ER homeostasis and neuronal death. Autophagy. 2020;16(3):531-47.

42. Li D, Qi J, Wang J, et al. Protective effect of dihydroartemisinin in inhibiting senescence of myeloid-derived suppressor cells from lupus mice via Nrf2/HO-1 pathway. Free Radic Biol Med. 2019;1(143):260-74.

43. Kaufmann SH, et al. The erasable Western blot. Anal Biochem. 1987;161:89-95.

44. Kaufmann SH, Kellner U. Erasure of Western blots after autoradiographic or chemiluminescent detection. In: Pound JD, editor. Immunochemical protocols. NJ.: Humana Press, Totowa; 1998. p. 223-35.

45. Pluvinage JV, Haney MS, Smith BAH, et al. CD22 blockade restores homeostatic microglial phagocytosis in ageing brains. Nature. 2019;568(7751):187-92.

46. Toller-Kawahisa JE, Canicoba NC, Venancio VP, et al. Systemic lupus erythematosus onset in lupus-prone B6.MRL/Ipr mice Is influenced by weight gain and Is preceded by an increase in neutrophil oxidative burst activity. Free Radic Biol Med. 2015;86:362-73.

47. Song J, et al. Circadian rhythm resynchronization improved isoflurane-induced cognitive dysfunction in aged mice. Exp Neurol. 2018;306:45-54

48. Xue YX, et al. Overexpression of protein kinase Mzeta in the Prelimbic Cortex enhances the formation of long-term fear memory. Neuropsychopharmacology. 2015;40(9):2146-56.

49. Song Y, et al. Green tea polyphenols improve isoflurane-induced cognitive impairment via modulating oxidative stress. J Nutr Biochem. 2019;73: 108213.

50. Anderson KJ, Miller KM, Fugaccia I, et al. Regional distribution of fluorojade $B$ staining in the hippocampus following traumatic brain injury. Exp Neurol. 2005;193(1):125-30.

51. Tomita M, Khan RL, Blehm BH, et al. The potential pathogenetic link between peripheral immune activation and the central innate immune response in neuropsychiatric systemic lupus erythematosus. Med Hypotheses. 2004;62(3):325-35.

52. Sakic B, Szechtman H, Denburg JA, et al. Progressive atrophy of pyramidal neuron dendrites in autoimmune MRL-Ipr mice. J Neuroimmunol. 1998;87(1-2):162-70.

53. Qiao X, Wang H, Lu L, et al. Hippocampal microglia CD40 mediates NPSLE cognitive dysfunction in mice. J Neuroimmunol. 2021;357: 577620 .
54. Gupta V, Bamezai RN. Human pyruvate kinase M2: a multifunctional protein. Protein science : a publication of the Protein Society. 2010:19(11):2031-2044.

55. Israelsen WJ, et al. PKM2 isoform-specific deletion reveals a differential requirement for pyruvate kinase in tumor cells. Cell. 2013;155:397-409.

56. Ashizaw K, Willinghan MC, Liang CM. In vivo regulation of monomertetramer conversion of pyruvate kinase subtype $\mathrm{M} 2$ by glucose is mediated via fructose 1,6-bisphosphate. J Biol Chem. 1991;266:16842-6.

57. Muñoz-Colmenero A, Fernández-Suárez A, Fatela-Cantillo D, et al. Plasma tumor M2-pyruvate kinase levels in different cancer types. Anticancer Res. 2015;35(7):4271-6.

58. Sominsky L, De Luca S, Spencer SJ. Microglia: key players in neurodevelopment and neuronal plasticity. Int J Biochem Cell Biol. 2018;94:56-60.

59. Morris GP, et al. Microglia: a new frontier for synaptic plasticity, learning and memory, and neurodegenerative disease research. Neurobiol Learn Mem. 2013;105:40-53.

60. Derecki NC, et al. Microglia as a critical player in both developmental and late-life CNS pathologies. Acta Neuropathol. 2014;128(3):333-45.

61. Jia L, Pina-Crespo J, Li Y. Restoring Wnt/beta-catenin signaling is a promising therapeutic strategy for Alzheimer's disease. Mol Brain. 2019;12(1):104.

62. Marchetti $B$, et al. Parkinson's disease, aging and adult neurogenesis: Wnt/beta-catenin signalling as the key to unlock the mystery of endogenous brain repair. Aging Cell. 2020;19(3): e13101.

63. Serafino A, et al. Targeting the Wnt/beta-catenin pathway in neurodegenerative diseases: recent approaches and current challenges. Expert Opin Drug Discov. 2020;1-19.

64. Jeong WJ, Ro EJ, Choi K-Y. Interaction between Wnt/ $\beta$-catenin and RAS-ERK pathways and an anti-cancer strategy via degradations of $\beta$-catenin and RAS by targeting the Wnt/ $\beta$-catenin pathway. NPJ Precis Oncol. 2018;2(1):5.

65. Duarte-Delgado NP, Vásquez G, Ortiz-Reyes BL. Blood-brain barrier disruption and neuroinflammation as pathophysiological mechanisms of the diffuse manifestations of neuropsychiatric systemic lupus erythematosus. Autoimmun Rev. 2019;18(4):426-32.

66. Dantzer R, O'Connor JC, Freund GG, et al. From inflammation to sickness and depression: when the immune system subjugates the brain. Nat Rev Neurosci. 2008:9:46-56.

67. Oitzl MS, van Oers H, Schöbitz B, et al. Interleukin-1 beta, but not interleukin-6, impairs spatial navigation learning. Brain Res. 1993;613:160-3.

68. Kothur K, Wienholt L, Brilot F, et al. CSF cytokines/chemokines as biomarkers in neuroinflammatory CNS disorders: a systematic review. Cytokine. 2016;77:227-37.

69. Yoshio T, Hirata D, Onda K, et al. Antiribosomal P protein antibodies in cerebrospinal fluid are associated with neuropsychiatric systemic lupus erythematosus. J Rheumatol. 2005;32(1):34-9.

70. Carter LM, Isenberg DA, Ehrenstein MR. Elevated serum BAFF levels are associated with rising anti-double-stranded DNA antibody levels and disease flare following B cell depletion therapy in systemic lupus erythematosus. Arthritis Rheum. 2013;65:2672-9.

71. Caro-Maldonado A, Wang R, Nichols AG, et al. Metabolic reprogramming is required for antibody production that is suppressed in anergic but exaggerated in chronically BAFF-exposed B cells. J Immunol. 2014;192:3626-36.

72. Yin Y, et al. Normalization of CD4+ T cell metabolism reverses lupus. Sci Transl Med. 2015;7:274ra18

73. Gergely PJ, et al. Mitochondrial hyperpolarization and ATP depletion in patients with systemic lupus erythematosus. Arthritis Rheum. 2002:46:175-90.

74. Huang N, Perl A. Metabolism as a target for modulation in autoimmune diseases. Trends Immunol. 2018;39(7):562-76.

75. Choi SC, Titov AA, Sivakumar R, et al. Immune cell metabolism in systemic lupus erythematosus. Curr Rheumatol Rep. 2016;18:66.

76. Komatsu N, Kodama K, Yamanouchi N, et al. Decreased regional cerebral metabolic rate for glucose in systemic lupus erythematosus patients with psychiatric symptoms. Eur Neurol. 1999;42:41-8.

77. Zheng $X$, Boyer $L$, Jin M, et al. Metabolic reprogramming during neuronal differentiation from aerobic glycolysis to neuronal oxidative phosphorylation. Elife. 2016;5: e13374.

78. Hinkle PC. P/O ratios of mitochondrial oxidative phosphorylation. Biochim Biophys Acta. 2005;1706:1-11. 
79. Goyal MS, Hawrylycz M, Miller JA, et al. Aerobic glycolysis in the human brain is associated with development and neotenous gene expression. Cell Metab. 2014;19(1):49-57.

80. Vander Heiden MG, Cantley LC, Thompson CB. Understanding the Warburg effect: the metabolic requirements of cell proliferation. Science. 2009;324(5930):1029-33.

81. Julia J, Jolivet R, Attwell D. Synaptic energy use and supply. Neuron. 2012;75(5):762-77.

82. Magistretti PJ, Allaman I. Lactate in the brain: from metabolic endproduct to signalling molecule. Nat Rev Neurosci. 2018;19(4):235-49.

83. Barros LF, Weber B. CrossTalk proposal: an important astrocyte-toneuron lactate shuttle couples neuronal activity to glucose utilisation in the brain. J Physiol. 2018;596(3):347-50.

84. Fernandez-Fernandez S, Almeida A, Bolaños JP. Antioxidant and bioenergetic coupling between neurons and astrocytes. Biochem J. 2012;443(1):3-11.

85. Krawczyk CM, Holowka T, Sun J, et al. Toll-like receptor-induced changes in glycolytic metabolism regulate dendritic cell activation. Blood. 2010;115(23):4742-9.

86. Haschemi A, Kosma P, Gille L, et al. The sedoheptulose kinase CARKL directs macrophage polarization through control of glucose metabolism. Cell Metab. 2012;15(6):813-26.

87. Xie M, et al. PKM2-dependent glycolysis promotes NLRP3 and AIM2 inflammasome activation. Nat Commun. 2016:25(7):13280.

88. Shirai T, et al. The glycolytic enzyme PKM2 bridges metabolic and inflammatory dysfunction in coronary artery disease. J Exp Med. 2016;213(3):337-54

89. Palsson-McDermott EM, et al. Pyruvate kinase $M 2$ regulates Hif-1alpha activity and IL-1 beta induction and is a critical determinant of the Warburg effect in LPS-activated macrophages. Cell Metab. 2015;21:65-80.

90. Ouyang X, et al. Digoxin suppresses pyruvate kinase M2-promoted HIF-1 a transactivation in steatohepatitis. Cell Metab. 2018;27(2):339350.e3.

91. Yang $\mathrm{W}$, et al. PKM2 phosphorylates histone $\mathrm{H} 3$ and promotes gene transcription and tumorigenesis. Cell. 2012;150(4):685-96.

92. Angiari $\mathrm{S}$, et al. Pharmacological activation of pyruvate kinase $\mathrm{M} 2$ inhibits CD4 + T cell pathogenicity and suppresses autoimmunity. Cell Metab. 2020;31(2):391-405.e8.

93. Yang $L$, et al. PKM2 regulates the Warburg effect and promotes HMGB1 release in sepsis. Nat Commun. 2014;14(5):4436.

94. Wu Q, Ge W, Chen Y, et al. PKM2 involved in neuronal apoptosis on hypoxic-ischemic encephalopathy in neonatal rats. Neurochem Res 2019;44(7):1602-12

95. Chen D, et al. Pyruvate kinase M2 increases angiogenesis, neurogenesis, and functional recovery mediated by upregulation of STAT3 and focal adhesion kinase activities after ischemic stroke in adult mice. Neurotherapeutics. 2018;15(3):770-84.

96. Dombrauckas JD, Santarsiero BD, Mesecar AD. Structural basis for tumor pyruvate kinase $\mathrm{M} 2$ allosteric regulation and catalysis. Biochemistry. 2005;44(27):9417-29.
97. Iqbal MA, et al. Pyruvate kinase $\mathrm{M} 2$ and cancer: an updated assessment. FEBS Lett. 2014;588:2685-92. https://doi.org/10.1016/j.febslet.2014.04 011.

98. Luo W, Semenza GL. Emerging roles of PKM2 in cell metabolism and cancer progression. Trends Endocrinol Metab. 2012;23:560-6.

99. Christofk H, Heiden MV, Harris M, Ramanathan A, Gerszten R. The M2 splice isoform of pyruvate kinase is important for cancer metabolism and tumour growth. Nature. 2008;452:230-3.

100. Israelsen WJ, Vander Heiden MG. Pyruvate kinase: function, regulation and role in cancer. Semin Cell Dev Biol. 2015;43:43-51.

101. Colonna M, Butovsky O. Microglia function in the central nervous system during health and neurodegeneration. Annu Rev Immunol. 2017:26(35):441-68.

102. Johnson RT, Richardson EP. The neurological manifestations of systemic lupus erythematosus. Medicine. 1968;47:337-69.

103. Brooks WM, Sibbitt WL Jr, Kornfeld M, et al. The histopathologic associates of neurometabolite abnormalities in fatal neuropsychiatric systemic lupus erythematosus. Arthritis Rheum. 2010;62:2055-63.

104. Mondal TK, Saha SK, Miller VM, et al. Autoantibody-mediated neuroinflammation: pathogenesis of neuropsychiatric systemic lupus erythematosus in the NZM88 murine model. Brain Behav Immun. 2008;22:949-59.

105. Crescenzi R, DeBrosse C, Nanga RPR, et al. Longitudinal imaging reveals sub-hippocampal dynamics in glutamate levels associated with histopathologic events in a mouse model of tauopathy and healthy mice. Hippocampus. 2017;27(3):285-302.

106. Jain S, LaFrancois JJ, Botterill JJ, et al. Adult neurogenesis in the mouse dentate gyrus protects the hippocampus from neuronal injury following severe seizures. Hippocampus. 2019;29(8):683-709.

107. Crupi R, Cambiaghi M, Spatz L, et al. Reduced adult neurogenesis and altered emotional behaviors in autoimmune-prone B-cell activating factor transgenic mice. Biol Psychiatry. 2010;67:558-66.

108. Bialas AR, et al. Microglia-dependent synapse loss in type I interferonmediated lupus. Nature. 2017;546(7659):539-43.

109. Jeltsch-David H, Muller S. Neuropsychiatric systemic lupus erythematosus and cognitive dysfunction: the MRL-Ipr mouse strain as a model. Autoimmun Rev. 2014;13(9):963-73.

110. Huerta PT, Kowal C, DeGiorgio LA, et al. Immunity and behavior: antibodies alter emotion. Proc Natl Acad Sci U S A. 2006;103(3):678-83.

111. Tang B, Matsuda T, Akira S, et al. Age-associated increase in interleukin 6 in MRL/Ipr mice. Int Immunol. 1991;3:273-8.

112. Dantzer R. Cytokine, sickness behavior, and depression. Immunol Allergy Clin North Am. 2009;29:247-64.

\section{Publisher's Note}

Springer Nature remains neutral with regard to jurisdictional claims in published maps and institutional affiliations.
Ready to submit your research? Choose BMC and benefit from:

- fast, convenient online submission

- thorough peer review by experienced researchers in your field

- rapid publication on acceptance

- support for research data, including large and complex data types

- gold Open Access which fosters wider collaboration and increased citations

- maximum visibility for your research: over 100M website views per year

At $\mathrm{BMC}$, research is always in progress.

Learn more biomedcentral.com/submissions 